\title{
APPROXIMATION OF SOLUTIONS OF HAMILTON-JACOBI EQUATIONS ON THE HEISENBERG GROUP
}

\author{
Yves Achdou $^{1,2}$ And Italo Capuzzo-Dolcetta ${ }^{3}$
}

\begin{abstract}
We propose and analyze numerical schemes for viscosity solutions of time-dependent Hamilton-Jacobi equations on the Heisenberg group. The main idea is to construct a grid compatible with the noncommutative group geometry. Under suitable assumptions on the data, the Hamiltonian and the parameters for the discrete first order scheme, we prove that the error between the viscosity solution computed at the grid nodes and the solution of the discrete problem behaves like $\sqrt{h}$ where $h$ is the mesh step. Such an estimate is similar to those available in the Euclidean geometrical setting. The theoretical results are tested numerically on some examples for which semi-analytical formulas for the computation of geodesics are known. Other simulations are presented, for both steady and unsteady problems.
\end{abstract}

Mathematics Subject Classification. 70H20, 35F25, 35H20, 49L25, 65M06, 65M15.

Received April 17, 2007.

Published online May 27, 2008.

\section{INTRODUCTION}

This paper is concerned with the approximation of solutions of the Cauchy problem for Hamilton-Jacobi equations of the form

$$
\begin{aligned}
\frac{\partial u}{\partial t}+\Phi(|\sigma(x) D u|) & =0, & & \text { in } \mathbb{R}^{3} \times(0, \infty), \\
u(x, 0) & =u_{0}(x), & & \text { in } \mathbb{R}^{3},
\end{aligned}
$$

where $|\cdot|$ denotes the standard Euclidean norm on $\mathbb{R}^{2}, \Phi$ is a scalar positive, continuous and convex function on $\mathbb{R}_{+}$and, for $x=\left(x_{1}, x_{2}, x_{3}\right) \in \mathbb{R}^{3}, \sigma(x)$ is the $2 \times 3$ matrix

$$
\sigma(x)=\left(\begin{array}{ccc}
1 & 0 & 2 x_{2} \\
0 & 1 & -2 x_{1}
\end{array}\right) .
$$

Problem (1.1) arises as the dynamic programming equation for the optimal control problem

$$
\inf \left\{u_{0}(X(t ; x, c(t)))+\int_{0}^{t} \Phi^{*}(r(s)) \mathrm{d} s:|c(\cdot)| \leq 1, r(t) \geq 0\right\}
$$

Keywords and phrases. Degenerate Hamilton-Jacobi equation, Heisenberg group, finite difference schemes, error estimates.

1 UFR Mathématiques, Université Paris 7, Case 7012, 75251 Paris Cedex 05, France.

2 Laboratoire Jacques-Louis Lions, Université Paris 6, 75252 Paris Cedex 05, France. achdou@math.jussieu.fr

3 Dipartimento di Matematica, Università Roma "La Sapienza", Piazzale A. Moro 2, 00185 Roma, Italy.

capuzzo@mat . uniroma1.it 
where $X(t ; x, c(t))$ is the trajectory of the control system

$$
\dot{X}(t)=\sigma^{T}(X(t)) r(t) c(t), X(0)=x ; \quad c(t) \in \mathbb{R}^{2},|c(t)| \leq 1, r(t) \geq 0
$$

( ${ }^{T}$ denotes matrix transposition) and $\Phi^{*}$ is the convex conjugate of $\Phi$, see [3].

Observe that the Lie bracket $\left[\left(1,0,2 x_{2}\right),\left(0,1,-2 x_{1}\right)\right]=-4(0,0,1)$. This relation holds at every point $x$ so that by Chow's connectivity theorem, see for example [6], any two points in $\mathbb{R}^{3}$ can be connected in finite time by some trajectory of (1.3).

On the other hand, $\operatorname{Det}\left(\sigma(\mathrm{x}) \sigma^{\mathrm{T}}(\mathrm{x})\right) \equiv 0$; for this reasons system (1.3) has been proposed in Brockett [8] as a prototype of symmetric system generating a singular (or sub-Riemannian) metric structure on $\mathbb{R}^{3}$. In Section 2 below we will discuss in detail some relations existing between system (1.3) and the group operations in the Heisenberg group $H=\left(\mathbb{R}^{3}, \oplus\right)$ where

$$
y \oplus x=\left(x_{1}+y_{1}, x_{2}+y_{2}, x_{3}+y_{3}+2\left(x_{1} y_{2}-x_{2} y_{1}\right)\right) .
$$

In light of the previous remark, equation (1.1) can also be interpreted in the framework of the level set approach [25] to propagation of fronts by normal velocity in the anisotropic space $H$. A further motivation for the present work comes from the paper [5] where the role of equation (1.1) in the analysis of the small time behavior of the fundamental solution of the heat equation

$$
\frac{\partial u}{\partial t}-\operatorname{Trace}\left(\sigma^{T}(x) \sigma(x) D^{2} u\right)=0
$$

on the Heisenberg group is analyzed.

A major difficulty in the theoretical and numerical analysis of problem (1.1) comes from the fact that the Hamiltonian $H(x, p)=\Phi(|\sigma(x) p|)$ is non coercive; indeed if $\Phi(0)=0$ then $H(x, p)=0$ for all $\left(x_{1}, x_{2}\right) \neq(0,0)$ and $p$ such that $\left(p_{1}, p_{2}\right)$ is orthogonal to $\left(x_{1}, x_{2}\right)$. Because of this lack of coerciveness, the viscosity solutions of (1.1) cannot be expected to be Lipschitz continuous and in fact are just 1/2- Hölder continuous. For the same reason, the methods of Crandall and Lions [14] for the error analysis of monotone consistent approximation schemes are not available in our case.

The purpose of the present paper is to propose and analyze finite difference schemes for the approximation of viscosity solutions of (1.1). The main idea is to construct a non uniform grid in $\mathbb{R}^{3}$ which is compatible with the non-commutative translations (1.4) in such a way that it inherits the geometrical properties of the Heisenberg group. More precisely, the grid nodes are chosen as

$$
\xi_{i, j, k}=\left(i h, j h,(4 k+2 i j) h^{2}\right)
$$

where $h>0$ is the grid step, and $i, j, k$ are integers. Such a grid, whose points form a discrete subgroup of Heisenberg group $H$, has been introduced in [1] for designing a finite difference scheme for the Dirichlet problem with the Kohn Laplacian: Trace $\left(\sigma^{T}(x) \sigma(x) D^{2}\right)$.

Once the grid is constructed, it becomes natural to implement in our model classical finite difference schemes for first order nonlinear PDE's, see [25,27] and to analyze the error between the viscosity solution of (1.1) computed at the grid nodes and the solution of the corresponding discrete problem. Let us mention that semiLagrangian methods $[9,13,17,18]$ and higher order schemes $[18,19,26]$ for Hamilton-Jacobi equations have been widely studied, see also $[4,22,23]$ for Hamilton-Jacobi-Bellman equations.

Our main result is that, under suitable assumptions on $\Phi$, the initial data $u_{0}$ and the parameters for the discrete scheme, the error between the viscosity solution computed at the grid nodes and the solution of the discrete problem is bounded by $C \sqrt{h}$, which is precisely the same optimal estimate obtained by Crandall and Lions in the coercive case. 
The proof makes use of some precise information, established in Section 2, on the regularity and on the expansion of the support of the viscosity solution of (1.1) which are deduced from the Hopf-Lax type representation formula

$$
u(x, t)=\min _{y \in \mathbb{R}^{3}}\left(u_{0}(y)+t \Phi^{*}\left(\frac{d(x ; y)}{t}\right)\right),
$$

where $d$ is the sub-Riemannian distance associated to system (1.3), see [11,24]. The proof relies also in an essential way on viscosity solutions comparison techniques inspired by [14] since, as mentioned above, the exact solution cannot expected to be smooth and even Lipschitz continuous.

In order to test the theoretical results, we use the above mentioned schemes for both (1.1) and the associated static eikonal equation satisfied by $x \rightarrow d(x, y)$, namely

$$
|\sigma(x) d(x, y)|=1, \quad x \in \mathbb{R}^{3} \backslash\{y\}, \quad d(y, y)=0 .
$$

In particular, we test the numerical method against the semi-analytical formulas provided by Beals et al. [5] for the Carnot-Carathéodory distance.

The numerical analysis below is done for (1.1) for simplicity but it holds for more general Hamiltonians, for example of the form $H(x, p)=a(x) \Phi(|\sigma(x) p|)$, where $\Phi$ is as above and $a$ is sufficiently regular positive function, bounded from below and above by positive constants. A numerical computation with such an Hamiltonian is presented in Section 7.1.

The results in the present paper certainly hold for the higher dimensional version of equation (1.1) where

$$
\sigma(x)=\left(\begin{array}{ccc}
I & 0 & 2 x^{\prime \prime} \\
0 & I & -2 x^{\prime}
\end{array}\right)
$$

with $x^{\prime}, x^{\prime \prime} \in \mathbb{R}^{n}$ and $x=\left(x^{\prime}, x^{\prime \prime}, x_{2 n+1}\right)$.

Similar methods, with appropriate changes in the choice of the grid, may work for even more general problems like

$$
\frac{\partial u}{\partial t}+\Phi\left(\sup _{q \in C(x)} q \cdot p\right)=0 \quad \text { in } \mathbb{R}^{n} \times(0, \infty), \quad u(x, 0)=u_{0}(x) \quad \text { in } \mathbb{R}^{n},
$$

where $x \rightarrow C(x) \subseteq \mathbb{R}^{N}$ is a Lipschitz continuous convex, compact set-valued mapping satisfying in addition a controllability condition such as

- there exist $\delta>0$, a natural number $m \leq n$ and for each $x \in \mathbb{R}^{n}$ an $m \times n$ smooth matrix $\sigma(x)$ with bounded Jacobian such that $\sigma(x) B_{\mathbb{R}^{m}}(0, \delta) \subseteq C(x)$ for all $x \in \mathbb{R}^{n}$;

- $\sigma(x)$ satisfies the Hörmander-Chow rank condition at some order $k$ at all points $x \in \mathbb{R}^{n}$.

In this framework, the associated control system $\dot{X}(t) \in C(X(t))$ induces on $\mathbb{R}^{n}$ a stratified Lie group structure as in the model case considered in the present paper. This generalization will be the object of further investigation by the authors; in view of this remark, the present work can be regarded as an attempt in the direction of numerical computations on nonlinear PDE's models on Lie groups, a topic on which very little has been done, at least to our present knowledge, see http://citeseer.ist.psu.edu/436569.html for an updated bibliography on numerical analysis on Lie groups.

\section{Basic facts on the Heisenberg group}

Let us start by recalling relevant properties of the Heisenberg group $H=\left(\mathbb{R}^{3}, \oplus\right)$, where

$$
y \oplus x=\left(x_{1}+y_{1}, x_{2}+y_{2}, x_{3}+y_{3}+2\left(x_{1} y_{2}-x_{2} y_{1}\right)\right) .
$$

It is obvious that, in general, $x \oplus y \neq y \oplus x$. Note that $x \oplus y=y \oplus x$ if and only if $x_{1} y_{2}-x_{2} y_{1}=0$. 
The operator $D_{H}$ commutes with left translations, i.e. for all $y \in \mathbb{R}^{3}$, calling $\tau_{y}^{L} u$ the function $x \mapsto u(y \oplus x)$,

$$
D_{H}\left(\tau_{y}^{L} u\right)=\tau_{y}^{L}\left(D_{H} u\right)
$$

On the contrary, calling $\tau_{y}^{R} u$ the function $x \mapsto u(x \oplus y)$,

$$
\left(D_{H}\left(\tau_{y}^{R} u\right)\right)(x)=\left(\tau_{y}^{R}\left(D_{H} u\right)\right)(x)+4\left(\left(\partial_{x_{3}} u\right)(x \oplus y)\right)\left(\begin{array}{r}
-y_{2} \\
y_{1}
\end{array}\right) .
$$

Let $\alpha$ be a nonnegative parameter, the dilation of $x$ by $\alpha$ is defined by

$$
\alpha \cdot x=\left(\alpha x_{1}, \alpha x_{2}, \alpha^{2} x_{3}\right) .
$$

One can verify that $\alpha \cdot(x \oplus y)=\alpha \cdot x \oplus \alpha \cdot y$.

The operator $D_{H}$ has the following behavior w.r.t. dilations: calling $u \circ \alpha$ the function $x \mapsto u(\alpha \cdot x)$, we have

$$
D_{H}(u \circ \alpha)=\alpha\left(D_{H} u\right) \circ \alpha .
$$

Observe that for all $x \in \mathbb{R}^{3}$ and $y=\left(y_{1}, y_{2}, 0\right)$, one has

$$
x \oplus t y=x(t),
$$

where $x(t)$ is the solution of the ordinary differential equation

$$
\left(\begin{array}{l}
\dot{x}_{1}(t) \\
\dot{x}_{2}(t) \\
\dot{x}_{3}(t)
\end{array}\right)=\left(\begin{array}{ll}
1 & 0 \\
0 & 1 \\
2 x_{2}(t) & -2 x_{1}(t)
\end{array}\right)\left(\begin{array}{l}
y_{1} \\
y_{2}
\end{array}\right),
$$

with the initial value $x(0)=x$.

For any fixed $y \in \mathbb{R}^{3}$, the stationary eikonal type problem

$$
\left|D_{H} w_{y}(x)\right|=1 \quad \text { in } \mathbb{R}^{3} \backslash\{y\}, \quad w_{y}(y)=0
$$

has a unique viscosity solution satisfying

$$
\begin{aligned}
& w_{y}(x) \geq 0, \quad \forall x, y \in \mathbb{R}^{3}, \quad \lim _{y} w_{y}(x)=+\infty, \\
& w_{y}(x)+w_{z}(y) \geq w_{z}(x), \quad \forall x, y, z \in \mathbb{R}^{3},
\end{aligned}
$$

see $[2,3]$, where $|\cdot|$ is the standard Euclidean norm in $\mathbb{R}^{2}$.

We use the notation $d(x ; y)=w_{y}(x)$ for the so-called Carnot-Carathéodory distance. It follows easily from the left invariance and homogeneity of $D_{H}$, see (2.1) and (2.3), that

$$
d(z \oplus x ; z \oplus y)=d(x ; y), \quad \text { and } \quad d(\alpha \cdot x ; \alpha \cdot y)=\alpha d(x ; y) .
$$

It is also well-known, see [6], that for any $R>0$ there exists a constant $K(R)>0$ such that

$$
d(x ; y) \leq K(R)|x-y|^{\frac{1}{2}} \quad \text { for all } \quad x, y \in \mathbb{R}^{3},|x-y| \leq R .
$$

We denote by $|\cdot|_{K}$ the Korànyi homogeneous norm in $\mathbb{R}^{3}$, which is naturally associated with the Heisenberg group:

$$
|x|_{K}=\left(\left(x_{1}^{2}+x_{2}^{2}\right)^{2}+x_{3}^{2}\right)^{\frac{1}{4}} .
$$


It is clear that $|x|_{K}=\sqrt{x_{1}^{2}+x_{2}^{2}}=|x|$ for any horizontal vector $x=\left(x_{1}, x_{2}, 0\right)$. Note also that for each $\alpha \in \mathbb{R}_{+}$ and $x, y \in \mathbb{R}^{3},|\alpha \cdot x|_{K}=\alpha|x|_{K}$ and $|-y \oplus x|_{K}=|-x \oplus y|_{K}$. It is proved in [21] that $(x, y) \mapsto|-y \oplus x|_{K}$ defines a metric in $\mathbb{R}^{3}$. It can be seen that $x \mapsto|-y \oplus x|_{K}$ is a viscosity subsolution of (2.5).

We also recall that there exist two positive constants $c_{1}<c_{2}$ such that

$$
c_{1}|-x \oplus y|_{K} \leq d(x ; y) \leq c_{2}|-x \oplus y|_{K},
$$

see [6]. For what follows, we define the Carnot-Carathéodory balls

$$
B_{C}(r)=\left\{x \in \mathbb{R}^{3}, d(x ; 0) \leq r\right\},
$$

and the Korànyi balls

$$
B_{K}(r)=\left\{x \in \mathbb{R}^{3},|x|_{K} \leq r\right\}
$$

We will say that $u$ is Lipschitz continuous w.r.t. the left translations with a constant $L$ if, for all $y \in \mathbb{R}^{3}$,

$$
\sup _{z \in \mathbb{R}^{3}}|u(y \oplus z)-u(z)| \leq L|y|_{K} .
$$

Similarly, $u$ is Lipschitz continuous w.r.t. the right translations with a constant $L$ if, for all $y \in \mathbb{R}^{3}$,

$$
\sup _{z \in \mathbb{R}^{3}}|u(z \oplus y)-u(z)| \leq L|y|_{K} .
$$

For example, for any real valued Lipschitz continuous function $\chi$ on $\mathbb{R}_{+}, x \mapsto \chi\left(|x|_{K}\right)$ is Lipschitz continuous w.r.t. right translations. Also, any bounded subsolution of $\left|D_{H} w\right| \leq 1$ in $\mathbb{R}^{3}$ is Lipschitz continuous w.r.t. right translations, see [7].

\section{Some Properties of the Viscosity solutions}

If the initial datum $u_{0}$ is bounded and continuous and $\Phi:[0,+\infty) \rightarrow \mathbb{R}$ is convex nondecreasing with $\Phi(0)=0, \lim _{p \rightarrow \infty} \Phi(p)=+\infty$, then, introducing the conjugate function

$$
\Phi^{*}(q)=\sup _{p \geq 0}(p q-\Phi(p)),
$$

the Hopf-Lax formula

$$
u(x, t)=\inf _{y \in \mathbb{R}^{3}}\left(u_{0}(y)+t \Phi^{*}\left(\frac{d(x ; y)}{t}\right)\right),
$$

see [10-12,24], provides the unique continuous viscosity solution of problem (1.1), see [15].

It is simple to verify that $\Phi^{*}$ is convex and nondecreasing with $\Phi(0)=0$.

In what follows, we make the following assumption on $\Phi$ :

Assumption 3.1. The function $\Phi$ is convex and nondecreasing, $\Phi(0)=0, \lim _{p \rightarrow \infty} \Phi(p)=+\infty$, and the conjugate function $\Phi^{*}$ is such that

$$
\lim _{q \rightarrow+\infty} \Phi^{*}(q) / q=+\infty .
$$

The assumption is fulfilled for example by $\Phi(p)=\frac{1}{\alpha} p^{\alpha}$ with $\alpha \geq 1$. If Assumption 3.1 holds, then

$$
u(x, t)=\min _{y \in \mathbb{R}^{3}}\left(u_{0}(y)+t \Phi^{*}\left(\frac{d(x ; y)}{t}\right)\right) .
$$

For each $t \geq 0$, let $S(t)$ be the time $t$ map associated with (1.1), i.e. $S(t) u_{0}(x)=u(x, t)$ where $u$ is the viscosity solution of (1.1). In the following proposition we summarize several useful properties of $S(t)$. 
Proposition 3.1. Let $\Phi$ satisfy Assumption 3.1. Then, for $u_{0}$ and $v_{0}$ continuous in $\mathbb{R}^{3}$,

1. $\left\|\left(S(t) u_{0}-S(t) v_{0}\right)^{+}\right\|_{\infty} \leq\left\|\left(u_{0}-v_{0}\right)^{+}\right\|_{\infty}$.

2. $\left\|S(t) u_{0}-S(t) v_{0}\right\|_{\infty} \leq\left\|u_{0}-v_{0}\right\|_{\infty}$.

3. $\inf _{\mathbb{R}^{3}} u_{0} \leq S(t) u_{0} \leq \sup _{\mathbb{R}^{3}} u_{0}$.

4. $\left\|\tau_{y}^{L}\left(S(t) u_{0}\right)-S(t) u_{0}\right\|_{\infty} \leq\left\|\tau_{y}^{L}\left(u_{0}\right)-u_{0}\right\|_{\infty}$.

5. If $u_{0}$ is Lipschitz continuous w.r.t. left translations with a constant $L_{1}$, then so is $S(t) u_{0}$.

6. $S(t+\tau) u_{0} \leq S(t) u_{0}, \forall \tau>0$.

7. If $u_{0}$ is Lipschitz continuous w.r.t. right translations with a constant $L$, then for $K=\Phi\left(\frac{L}{c_{1}}\right)$, where $c_{1}$ appears in (2.9),

$$
\left\|S(t) u_{0}-S\left(t^{\prime}\right) u_{0}\right\| \leq K\left|t-t^{\prime}\right|, \quad \forall t, t^{\prime} \geq 0 .
$$

8. If $\operatorname{supp}\left(u_{0}\right) \subset B_{C}\left(R_{0}\right)$, then $S(t) u_{0}$ is compactly supported and there exists a function $R: \mathbb{R}_{+} \rightarrow \mathbb{R}_{+}$, nondecreasing, which only depends on $\Phi^{*}$ and on $\left\|u_{0}^{-}\right\|_{\infty}$, such that

$$
\operatorname{supp}\left(S(t) u_{0}\right) \subset B_{C}\left(R_{0}+R(t)\right) \subset B_{K}\left(\frac{1}{c_{1}}\left(R_{0}+R(t)\right)\right) .
$$

9. If

- $u_{0}$ is supported in the Carnot-Carathéodory ball $B_{C}\left(R_{0}\right)$;

- $u_{0}$ is Lipschitz continuous w.r.t left translations with a constant $L_{1}$;

- there exists $L_{2}$ such that $\left\|u_{0}\left(\cdot \oplus \delta e_{3}\right)-u_{0}(\cdot)\right\| \leq L_{2}|\delta|$, for all $\delta>0$,

then $S(t) u_{0}$ is Lipschitz continuous w.r.t. right translations with a constant

$$
L(t)=L_{1}+\frac{4 L_{2}\left(R_{0}+R(t)\right)}{c_{1}}
$$

Proof. To prove points 1 and 2, set $v(x, t)=S(t) v_{0}, u(x, t)=S(t) u_{0}$ and from (3.3), let $\bar{y}$ be such that $v(x, t)=v_{0}(\bar{y})+t \Phi^{*}\left(\frac{d(x ; \bar{y})}{t}\right)$.

Then,

$$
u(x, t)-v(x, t) \leq u_{0}(\bar{y})+t \Phi^{*}\left(\frac{d(x ; \bar{y})}{t}\right)-v_{0}(\bar{y})-t \Phi^{*}\left(\frac{d(x ; \bar{y})}{t}\right) .
$$

The above gives

$$
u(x, t)-v(x, t) \leq\left(u_{0}-v_{0}\right)(\bar{y}) \leq\left\|u_{0}-v_{0}\right\|_{\infty},
$$

and also

$$
u(x, t)-v(x, t) \leq\left(u_{0}-v_{0}\right)^{+}(\bar{y})
$$

From the above, it follows that

$$
(u-v)^{+}(x, t) \leq\left(u_{0}-v_{0}\right)^{+}(\bar{y}) \leq\left\|\left(u_{0}-v_{0}\right)^{+}\right\|_{\infty} .
$$

The proof of points 1 and 2 is now completed by exchanging the roles of $u$ and $v$.

To verify the right-hand side inequality at point 3 it is enough to take $y=x$ in the representation formula (3.3); on the other hand, since $\Phi^{*} \geq 0$ we have that

$$
u_{0}(y)+t \Phi^{*}\left(\frac{d(x ; y)}{t}\right) \geq \inf _{y \in \mathbb{R}^{3}} u_{0},
$$

which implies the left-hand side inequality at point 3 . 
Point 4 stems from point 2 and from the fact that $\tau_{y}^{L}\left(S(t) u_{0}\right)=S(t)\left(\tau_{y}^{L} u_{0}\right)$. The last identity comes from (3.3) and (2.6), because

$$
\begin{aligned}
\left(\tau_{y}^{L}\left(S(t) u_{0}\right)\right)(x)=u(y \oplus x, t) & =\min _{z \in \mathbb{R}^{3}}\left(u_{0}(z)+t \Phi^{*}\left(\frac{d(y \oplus x ; z)}{t}\right)\right) \\
& =\min _{z^{\prime} \in \mathbb{R}^{3}}\left(u_{0}\left(y \oplus z^{\prime}\right)+t \Phi^{*}\left(\frac{d\left(y \oplus x ; y \oplus z^{\prime}\right)}{t}\right)\right) \\
& =\min _{z^{\prime} \in \mathbb{R}^{3}}\left(u_{0}\left(y \oplus z^{\prime}\right)+t \Phi^{*}\left(\frac{d\left(x ; z^{\prime}\right)}{t}\right)\right) \\
& =\min _{z^{\prime} \in \mathbb{R}^{3}}\left(\tau_{y}^{L} u_{0}\left(z^{\prime}\right)+t \Phi^{*}\left(\frac{d\left(x ; z^{\prime}\right)}{t}\right)\right) \\
& =\left(S(t)\left(\tau_{y}^{L} u_{0}\right)\right)(x) .
\end{aligned}
$$

Point 5 is an immediate consequence of point 4 .

For proving point 6 , observe that since $\Phi \geq 0$,

$$
(t+\tau) \Phi^{*}\left(\frac{d(x ; y)}{t+\tau}\right)=\sup _{p \geq 0}(p d(x ; y)-(t+\tau) \Phi(p)) \leq \sup _{p \geq 0}(p d(x ; y)-t \Phi(p))=t \Phi^{*}\left(\frac{d(x ; y)}{t}\right),
$$

and the claim follows from the Hopf-Lax formula.

Let us prove point 7: let $\bar{y}=\bar{y}(x, t)$ be such that $u(x, t)=u_{0}(\bar{y})+t \Phi^{*}(d(x ; \bar{y}) / t)$. Then, by $(3.3)$,

$$
u\left(x, t^{\prime}\right)-u(x, t) \leq u_{0}(y)+t^{\prime} \Phi^{*}\left(\frac{d(x ; y)}{t^{\prime}}\right)-u_{0}(\bar{y})-t \Phi^{*}\left(\frac{d(x ; \bar{y})}{t}\right), \quad \forall y \in \mathbb{R}^{3} .
$$

Using now the Lipschitz continuity w.r.t. right translations and (2.9), we obtain from (3.7)

$$
u\left(x, t^{\prime}\right)-u(x, t) \leq \frac{L}{c_{1}} d(y ; \bar{y})+\left(t^{\prime}-t\right) \Phi^{*}\left(\frac{d(x ; \bar{y})}{t}\right) .
$$

With no restriction, we can assume that $t^{\prime}<t$. Consider the geodesic connecting $x$ to $\bar{y}$, and choose $y$ on the geodesic such that

$$
d(x ; y) / t^{\prime}=d(x ; \bar{y}) / t .
$$

It is clear that $d(x ; \bar{y})=d(x ; y)+d(y ; \bar{y})$. Thus, from (3.8),

$$
\begin{aligned}
u\left(x, t^{\prime}\right)-u(x, t) & \leq \frac{L}{c_{1}}(d(x ; \bar{y})-d(x, y))+\left(t^{\prime}-t\right) \Phi^{*}\left(\frac{d(x ; \bar{y})}{t}\right) \\
& =\left(t-t^{\prime}\right)\left(\frac{L}{c_{1}} \frac{d(x ; \bar{y})}{t}-\Phi^{*}\left(\frac{d(x ; \bar{y})}{t}\right)\right) \\
& \leq\left(t-t^{\prime}\right) \Phi\left(\frac{L}{c_{1}}\right),
\end{aligned}
$$

where the second line comes from (3.9), and the third line comes from Fenchel's inequality.

For the proof of point 8 , assume that $u_{0}$ is supported in the Carnot-Carathéodory ball $B_{C}\left(R_{0}\right)$. We are going to use the representation formula (3.1) to prove that for each $t>0, x \mapsto u(x, t)$ has a bounded support. We first observe that $\Phi^{*}$ is a nonnegative function. We proceed in two steps:

First step. From (3.2), we see that there exists a positive number $\xi$ such that

$$
\Phi^{*}(q) \geq q\left\|u_{0}^{-}\right\|_{\infty}, \quad \forall q \geq \xi .
$$


If $d(x ; 0)>R_{0}+\max (1, \xi t)$, then for all $y \in \operatorname{supp}\left(u_{0}\right)$, we have $d(x ; y) \geq d(x ; 0)-d(y ; 0)>\max (1, \xi t)$. Thus, from (3.10), $u_{0}(y)+t \Phi^{*}(d(x ; y) / t) \geq u_{0}(y)+\left\|u_{0}^{-}\right\|_{\infty} \geq 0$. Note also that for $y \notin \operatorname{supp}\left(u_{0}\right), u_{0}(y)+$ $t \Phi^{*}(d(x ; y) / t) \geq 0$ because $\Phi^{*}$ is nonnegative. This and the representation formula (3.3) imply that if $d(x ; 0)>$ $R_{0}+\max (1, \xi t)$, then $u(x, t) \geq 0$.

Second step. If $d(x ; 0)>R_{0}+\max (1, \xi t)$, take a sequence $y_{n} \notin \operatorname{supp}\left(u_{0}\right)$ such that $\lim _{n \rightarrow \infty} y_{n}=x$. We have

$$
0 \leq u(x, t) \leq u_{0}\left(y_{n}\right)+t \Phi^{*}\left(\frac{d\left(x ; y_{n}\right)}{t}\right)=t \Phi^{*}\left(\frac{d\left(x ; y_{n}\right)}{t}\right) .
$$

We have $\lim _{n \rightarrow \infty} d\left(x ; y_{n}\right)=0$ thanks to $(2.7)$, which yields $u(x, t)=0$ since $\Phi^{*}(0)=0$.

Point 8 is proved with $R(t)=\max (1, \xi t)$.

To prove point 9 , we see that

$$
u(x \oplus y, t)-u(x, t)=u(y \oplus x, t)-u(x, t)+u(x \oplus y, t)-u(y \oplus x, t) .
$$

But $x \oplus y=4\left(x_{2} y_{1}-x_{1} y_{2}\right) e_{3} \oplus y \oplus x$. Therefore,

$$
|u(x \oplus y, t)-u(x, t)| \leq|u(y \oplus x, t)-u(x, t)|+\left|u\left(4\left(x_{2} y_{1}-x_{1} y_{2}\right) e_{3} \oplus y \oplus x, t\right)-u(y \oplus x)\right| .
$$

We make out two cases:

(1) If $\sqrt{\left(x_{1}+y_{1}\right)^{2}+\left(x_{2}+y_{2}\right)^{2}}>\frac{1}{c_{1}}\left(R_{0}+R(t)\right)$, then $u(x \oplus y, t)=u(y \oplus x, t)=0$ and we have

$$
|u(x \oplus y, t)-u(x, t)|=|u(y \oplus x, t)-u(x, t)| \leq L_{1}|y|_{K},
$$

from point 5 .

(2) If $\sqrt{\left(x_{1}+y_{1}\right)^{2}+\left(x_{2}+y_{2}\right)^{2}} \leq \frac{1}{c_{1}}\left(R_{0}+R(t)\right)$, then

$$
\left|x_{2} y_{1}-x_{1} y_{2}\right|=\left|\left(x_{2}+y_{2}\right) y_{1}-\left(x_{1}+y_{1}\right) y_{2}\right| \leq \sqrt{\left(x_{1}+y_{1}\right)^{2}+\left(x_{2}+y_{2}\right)^{2}}|y|_{K} \leq \frac{1}{c_{1}}\left(R_{0}+R(t)\right)|y|_{K}
$$

and we have that

$$
\begin{aligned}
|u(x \oplus y, t)-u(x, t)| & \leq|u(y \oplus x, t)-u(x, t)|+\left|u\left(4\left(x_{2} y_{1}-x_{1} y_{2}\right) e_{3} \oplus y \oplus x, t\right)-u(y \oplus x)\right| \\
& \leq\left\|\tau_{y}^{L}\left(u_{0}\right)-u_{0}\right\|_{\infty}+\left\|\tau_{4\left(x_{2} y_{1}-x_{1} y_{2}\right) e_{3}}^{L}\left(u_{0}\right)-u_{0}\right\|_{\infty} \\
& \leq L_{1}|y|_{K}+4 L_{2}\left|x_{2} y_{1}-x_{1} y_{2}\right| \\
& \leq\left(L_{1}+\frac{4 L_{2}\left(R_{0}+R(t)\right)}{c_{1}}\right)|y|_{K},
\end{aligned}
$$

where we have used successively point 4 , the definitions of $L_{1}$ and $L_{2}$ and the estimate on $\left|x_{2} y_{1}-x_{1} y_{2}\right|$ obtained just above.

Thus $S(t) u_{0}$ is Lipschitz continuous w.r.t. right translations with the constant $L(t)$ in (3.6).

Remark 3.1. In Proposition 3.1, the assumptions of point 9 imply the fact that $u_{0}$ is Lipschitz continuous w.r.t. right translations with a constant $L=L_{1}+4 \frac{L_{2} R}{c_{1}}$. Therefore the assumptions of point 9 imply point 7 with

$$
K=\Phi\left(L_{1}+4 \frac{L_{2} R}{c_{1}}\right)
$$

Remark 3.2. Note that any compactly supported function $u_{0}$ which is Lipschitz continuous (with the standard definition of Lipschitz continuity in $\mathbb{R}^{3}$ ) satisfies all the assumptions in Proposition 3.1. 


\section{Finite DiffEREnCE SCHEMES}

\subsection{Monotone schemes on a discrete subgroup of $\boldsymbol{H}$}

Let $T$ be a positive time. We are interested in approximating $u$ for times $t \leq T$. Let $P$ be a positive integer and $\Delta t=\frac{T}{P}$. Let $h$ be a positive real number. Hereafter, we assume that there exists a constant $C$ such that

$$
\Delta t \leq C h .
$$

For three integers $i, j, k$ we define the nodes $\xi_{i, j, k}=\left(i h, j h,(4 k+2 i j) h^{2}\right)$, and for a nonnegative integer $n$, we define $t_{n}=n \Delta t$. This lattice was first introduced in [1], as the key ingredient for a second order finite difference scheme for the Kohn Laplacian on the Heisenberg group. Calling $\left(e_{1}, e_{2}, e_{3}\right)$ the canonical basis of $\mathbb{R}^{3}$, we have

$$
\begin{gathered}
\xi_{i, j, k} \oplus \pm h e_{1}=\xi_{i \pm 1, j, k}, \\
\xi_{i, j, k} \oplus \pm h e_{2}=\xi_{i, j \pm 1, k \mp i} .
\end{gathered}
$$

More generally,

$$
\xi_{\ell, m, n} \oplus \xi_{i, j, k}=\xi_{\ell+i, m+j, k+n-j \ell} .
$$

Formulas (4.2) and (4.3) clearly show the relationship between the grid and the group operations $\oplus$ and $\cdot$. Since $\xi_{i, j, k} \oplus \xi_{\ell, m, n}=\xi_{\ell+i, m+j, k+n-i m}$, we see that $\xi_{i, j, k} \oplus \xi_{\ell, m, n}$ and $\xi_{\ell, m, n} \oplus \xi_{i, j, k}$ coincide if and only if $i m=j \ell$.

Capital letters $U, V, \ldots$ will stand for discrete functions defined on the lattice $\left\{\xi_{i, j, k}, i, j, k \in \mathbb{Z}\right\}$ and their values at $\xi_{i, j, k}$ will be written $U_{i, j, k}, V_{i, j, k}, \ldots$ The notations $\Delta_{+}^{1} U$ and $\Delta_{+}^{2} U$ will be used for the discrete functions:

$$
\left(\Delta_{+}^{1} U\right)_{i, j, k}=U_{i+1, j, k}-U_{i, j, k}, \quad\left(\Delta_{+}^{2} U\right)_{i, j, k}=U_{i, j+1, k-i}-U_{i, j, k} .
$$

The value of the numerical approximation of $u\left(\xi_{i, j, k}, t_{n}\right)$ will be written $U_{i, j, k}^{n}$.

We will consider numerical schemes

$$
U_{i, j, k}^{n+1}=G\left(U_{i, j, k}^{n}, U_{i+1, j, k}^{n}, U_{i-1, j, k}^{n}, U_{i, j+1, k-i}^{n}, U_{i, j-1, k+i}^{n}\right),
$$

such that there exists a continuous function $g: \mathbb{R}^{4} \rightarrow \mathbb{R}$, called the numerical Hamiltonian, with

$$
\begin{aligned}
& G\left(U_{i, j, k}, U_{i+1, j, k}, U_{i-1, j, k}, U_{i, j+1, k-i}, U_{i, j-1, k+i}\right)= \\
& \quad U_{i, j, k}-\Delta t g\left(\frac{1}{h}\left(\Delta_{+}^{1} U\right)_{i, j, k}, \frac{1}{h}\left(\Delta_{+}^{1} U\right)_{i-1, j, k}, \frac{1}{h}\left(\Delta_{+}^{2} U\right)_{i, j, k}, \frac{1}{h}\left(\Delta_{+}^{2} U\right)_{i, j-1, k+i}\right) .
\end{aligned}
$$

For the scheme (4.4) to be consistent with the Hamilton-Jacobi equation, we must have

$$
g(a, a, b, b)=\Phi\left(\left|\left(\begin{array}{l}
a \\
b
\end{array}\right)\right|\right)
$$

We will say that (4.4) is monotone if $G$ is a nondecreasing function of each of its five arguments. We will say that (4.4) is monotone on $[-R, R]$ if $G\left(U_{i, j, k}, U_{i+1, j, k}, U_{i-1, j, k}, U_{i, j+1, k-i}, U_{i, j-1, k+i}\right)$ is a nondecreasing function of each of its five arguments as long as $\left(\Delta_{+}^{1} U\right)_{i, j, k},\left(\Delta_{+}^{1} U\right)_{i-1, j, k},\left(\Delta_{+}^{2} U\right)_{i, j, k}$ and $\left(\Delta_{+}^{2} U\right)_{i, j-1, k+i}$ are contained in $[-R, R]$.

For brevity, we will use the notation $\vec{G}(U)=\left(G(U)_{i, j, k}\right)_{i, j, k \in \mathbb{Z}}$. We will also use the notation $\|U\|_{\infty}=$ $\sup _{i, j, k \in \mathbb{Z}}\left|U_{i, j, k}\right|$. We will say that $U \in \ell^{\infty}\left(\mathbb{Z}^{3}\right)$ if $\|U\|_{\infty}<+\infty$.

For $\Lambda>0$, we call $\mathcal{C}_{\Lambda}$ the set

$$
\mathcal{C}_{\Lambda}=\left\{U \in \ell^{\infty}\left(\mathbb{Z}^{3}\right),\left|\left(\Delta_{+}^{1} U\right)_{i, j, k}\right|<\Lambda h,\left|\left(\Delta_{+}^{2} U\right)_{i, j, k}\right|<\Lambda h, \forall i, j, k \in \mathbb{Z}\right\} .
$$


Finally, for $(\ell, m, n) \in \mathbb{Z}^{3}$, we note $\tau_{(\ell, m, n)}^{L} U$ the discrete function defined by

$$
\left(\tau_{(\ell, m, n)}^{L} U\right)_{i, j, k}=U_{\ell+i, m+j, k+n-4 j \ell} .
$$

Proposition 4.1. Assume that the scheme (4.4) is consistent and monotone on $[-\Lambda, \Lambda]$. Then

1. Identifying $\lambda \in \mathbb{R}$ with the constant function $\lambda$ on $\mathbb{Z}^{3}$, we have $\vec{G}(U+\lambda)=\vec{G}(U)+\lambda$, for all discrete function $U$.

2. For $U$ and $V$ in $\mathcal{C}_{\Lambda}$,

$$
\left\|(\vec{G}(U)-\vec{G}(V))^{+}\right\|_{\infty} \leq\left\|(U-V)^{+}\right\|_{\infty} .
$$

3. For $U$ and $V$ in $\mathcal{C}_{\Lambda}$ such that $U \leq V, \vec{G}(U) \leq \vec{G}(V)$.

4. For $U$ and $V$ in $\mathcal{C}_{\Lambda}$,

$$
\|\vec{G}(U)-\vec{G}(V)\|_{\infty} \leq\|U-V\|_{\infty} .
$$

5. The operator $\vec{G}$ commutes with the left lattice translations: for $(\ell, m, n) \in \mathbb{Z}^{3}$,

$$
\vec{G}\left(\tau_{(\ell, m, n)}^{L} U\right)=\tau_{(\ell, m, n)}^{L} \vec{G}(U) .
$$

6. If $U^{0} \in \mathcal{C}_{\Lambda}$ and if there exists a positive number $L_{1}$ such that for all $(\ell, m, n) \in \mathbb{Z}^{3},\left\|\tau_{(\ell, m, n)}^{L} U^{0}-U^{0}\right\|_{\infty} \leq$ $L_{1}\left|\xi_{\ell, m, n}\right|_{K}$, then for all $p \geq 0, U^{p}=\vec{G}^{p}\left(U^{0}\right)$ has the same property.

7. If the discrete function $U^{0}$ satisfies: there exist two positive integers $I_{0}$ and $J_{0}$ and two positive real numbers $L_{1}$ and $L_{2}$ such that

- $U_{i, j, k}^{0}=0$ if $|i|>I_{0}$ and $|j|>J_{0}$;

- for all $(\ell, m, n) \in \mathbb{Z}^{3},\left\|\tau_{(\ell, m, n)}^{L} U^{0}-U^{0}\right\|_{\infty} \leq L_{1}\left|\xi_{\ell, m, n}\right|_{K}$;

- for all $k \in \mathbb{Z},\left\|\tau_{(0,0, k)}^{L} U^{0}-U^{0}\right\|_{\infty} \leq 4 L_{2}|k| h^{2}$;

- $L_{1}+4 L_{2}\left(P+\max \left(I_{0}, J_{0}\right)\right) h<\Lambda$,

then for all $p \geq 0, U^{p}=\vec{G}^{p}\left(U^{0}\right)$ is such that

$$
\begin{aligned}
\left\|\Delta_{+}^{1} U^{p}\right\|_{\infty} & \leq\left(L_{1}+4 L_{2}\left(p+J_{0}\right) h\right) h, \\
\left\|\Delta_{+}^{2} U^{p}\right\|_{\infty} & \leq\left(L_{1}+4 L_{2}\left(p+I_{0}\right) h\right) h .
\end{aligned}
$$

8. Under the assumptions of point 7 on $U^{0}$, there exists a constant $K^{\prime}$ depending on $L_{1}, L_{2}, P h,\left(I_{0}+J_{0}\right) h$ such that, for all $p<P$,

$$
\left\|U^{p+1}-U^{p}\right\|_{\infty} \leq K^{\prime} \Delta t
$$

Proof. Point 1 is a direct consequence of (4.5).

If $V \in \mathcal{C}_{\Lambda}$, then for all constant $\alpha, V+\alpha \in \mathcal{C}_{\Lambda}$. Thus, if the two lattice functions $U$ and $V$ belong to $\mathcal{C}_{\Lambda}$, then $U$ and $V+\left\|(U-V)^{+}\right\|_{\infty}$ belong to $\mathcal{C}_{\Lambda}$. From this, the mononicity of $G$, and the inequality $U_{i, j, k} \leq V_{i, j, k}+\left\|(U-V)^{+}\right\|_{\infty}$, for all $(i, j, k) \in \mathbb{Z}^{3}$, we deduce that $\vec{G}(U) \leq \vec{G}(V)+\left\|(U-V)^{+}\right\|_{\infty}$. This implies (4.8).

Point 3 is straightforward consequence of (4.8). Also from (4.8), we see that

$$
\begin{aligned}
\|\vec{G}(U)-\vec{G}(V)\|_{\infty} & =\left\|(\vec{G}(U)-\vec{G}(V))^{+}-(\vec{G}(U)-\vec{G}(V))^{-}\right\|_{\infty} \\
& =\max \left(\left\|(\vec{G}(U)-\vec{G}(V))^{+}\right\|_{\infty},\left\|(\vec{G}(V)-\vec{G}(U))^{+}\right\|_{\infty}\right) \\
& \leq \max \left(\left\|(U-V)^{+}\right\|_{\infty},\left\|(V-U)^{+}\right\|_{\infty}\right) \\
& =\|U-V\|_{\infty},
\end{aligned}
$$

and we have proved (4.9).

Identity (4.10) comes from straightforward calculus. 
We have $\left\|\tau_{(\ell, m, n)}^{L} \vec{G}\left(U^{0}\right)-\vec{G}\left(U^{0}\right)\right\|_{\infty}=\left\|\vec{G}\left(\tau_{(\ell, m, n)}^{L} U^{0}\right)-\vec{G}\left(U^{0}\right)\right\|_{\infty}$ from (4.10). It is simple to verify that if $U^{0}$ belongs to $\mathcal{C}_{\Lambda}$, then, for all $(\ell, m, n) \in \mathbb{Z}^{3}, \tau_{(\ell, m, n)}^{L} U^{0} \in \mathcal{C}_{\Lambda}$. Thus, we can use (4.9), and we obtain that

$$
\left\|\tau_{(\ell, m, n)}^{L} \vec{G}\left(U^{0}\right)-\vec{G}\left(U^{0}\right)\right\|_{\infty} \leq\left\|\tau_{(\ell, m, n)}^{L} U^{0}-U^{0}\right\|_{\infty} \leq L_{1}\left|\xi_{\ell, m, n}\right|_{K}
$$

This proves point 6 for $p=1$. For $p>1$, we proceed by induction.

To prove point 7 , we first observe that $U^{0}$ belongs to $\mathcal{C}_{\Lambda}$, because

$$
\begin{aligned}
&\left(\Delta_{+}^{1} U^{0}\right)_{i, j, k}=U_{i+1, j, k}^{0}-U_{i, j, k}^{0}=U_{i+1, j, k}^{0}-U_{i+1, j, k-j}^{0}+U_{i+1, j, k-j}^{0}-U_{i, j, k}^{0}, \\
&\left(\Delta_{+}^{2} U^{0}\right)_{i, j, k}=U_{i, j+1, k-i}^{0}-U_{i, j, k}^{0}=U_{i, j+1, k-i}^{0}-U_{i, j+1, k}^{0}+U_{i, j+1, k}^{0}-U_{i, j, k}^{0} .
\end{aligned}
$$

Moreover, if $|i|>I_{0}$, then $U_{i, j+1, k-i}^{0}-U_{i, j+1, k}^{0}=0$, and if $|j|>J_{0}, U_{i+1, j, k}^{0}-U_{i+1, j, k-j}^{0}=0$. This, together with the other assumptions on $U^{0}$ imply that $\left|U_{i, j+1, k-i}^{0}-U_{i, j+1, k}^{0}\right| \leq 4 L_{2}|i| h^{2} \leq 4 L_{2} I_{0} h^{2}$. Similarly, $\mid U_{i+1, j, k}^{0}-$ $U_{i+1, j, k-j}^{0} \mid \leq 4 L_{2} J_{0} h^{2}$. Therefore

$$
\begin{aligned}
& \left|\left(\Delta_{+}^{1} U^{0}\right)_{i, j, k}\right| \leq\left|U_{i+1, j, k}^{0}-U_{i+1, j, k-j}^{0}\right|+\left|U_{i+1, j, k-j}^{0}-U_{i, j, k}^{0}\right| \leq\left(L_{1}+4 L_{2} J_{0} h\right) h<\Lambda h, \\
& \left|\left(\Delta_{+}^{2} U^{0}\right)_{i, j, k}\right| \leq\left|U_{i, j+1, k-i}^{0}-U_{i, j+1, k}^{0}\right|+\left|U_{i, j+1, k}^{0}-U_{i, j, k}^{0}\right| \leq\left(L_{1}+4 L_{2} I_{0} h\right) h<\Lambda h .
\end{aligned}
$$

Assume now that for some $q, 0 \leq q<P$, (4.11) is true for all $p, 0 \leq p \leq q$. Then, for $p \leq q, U^{p} \in \mathcal{C}_{\Lambda}$ because $L_{1}+4 L_{2}\left(P+\max \left(I_{0}, J_{0}\right)\right) h<\Lambda$. We also verify that $U_{i, j, k}^{p}=0$ if $|i|>I_{0}+p$ or if $|j|>J_{0}+p$. Moreover, we know from points 5 and 4 that

$$
\begin{gathered}
\left\|\tau_{(\ell, m, n)}^{L} U^{p}-U^{p}\right\|_{\infty} \leq L_{1}\left|\xi_{\ell, m, n}\right|, \quad \forall(\ell, m, n) \in \mathbb{Z}^{3}, \\
\left\|\tau_{(0,0, n)}^{L} U^{p}-U^{p}\right\|_{\infty} \leq 4 L_{2}|n| h^{2}, \quad \forall n \in \mathbb{Z} .
\end{gathered}
$$

We wish to study the properties of $U^{q+1}=\vec{G}\left(U^{q}\right)$ : we see immediately that $U_{i, j, k}^{q+1}=0$ if $|i|>I_{0}+q+1$ or if $|j|>J_{0}+q+1$ and we deduce from (4.9)-(4.10) and from $U^{q} \in \mathcal{C}_{\Lambda}$ that

$$
\begin{gathered}
\left\|\tau_{(\ell, m, n)}^{L} U^{q+1}-U^{q+1}\right\|_{\infty} \leq L_{1}\left|\xi_{\ell, m, n}\right|, \quad \forall(\ell, m, n) \in \mathbb{Z}^{3}, \\
\left\|\tau_{(0,0, n)}^{L} U^{q+1}-U^{q+1}\right\|_{\infty} \leq 4 L_{2}|n| h^{2}, \quad \forall n \in \mathbb{Z} .
\end{gathered}
$$

Now, we use exactly the same arguments as those we just used for $U^{0}$ and prove that

$$
\begin{aligned}
& \left|\left(\Delta_{+}^{1} U^{q+1}\right)_{i, j, k}\right| \leq\left|U_{i+1, j, k}^{q+1}-U_{i+1, j, k-j}^{q+1}\right|+\left|U_{i+1, j, k-j}^{q+1}-U_{i, j, k}^{q+1}\right| \leq\left(L_{1}+4 L_{2}\left(J_{0}+q+1\right) h\right) h<\Lambda h, \\
& \left|\left(\Delta_{+}^{2} U^{q+1}\right)_{i, j, k}\right| \leq\left|U_{i, j+1, k-i}^{q+1}-U_{i, j+1, k}^{q+1}\right|+\left|U_{i, j+1, k}^{q+1}-U_{i, j, k}^{q+1}\right| \leq\left(L_{1}+4 L_{2}\left(I_{0}+q+1\right) h\right) h<\Lambda h .
\end{aligned}
$$

We have proved (4.11) by induction.

For proving the last point, we call $\tilde{\Lambda}=\left(L_{1}+4 L_{2}\left(\max \left(I_{0}, J_{0}\right)+P\right) h\right)$ : we have $\tilde{\Lambda}<\Lambda$; from (4.11) and from the monotonicity of the scheme, we see that

$$
\begin{aligned}
& G\left(U_{i, j, k}^{p}, U_{i, j, k}^{p}-\tilde{\Lambda} h, U_{i, j, k}^{p}-\tilde{\Lambda} h, U_{i, j, k}^{p}-\tilde{\Lambda} h, U_{i, j, k}^{p}-\tilde{\Lambda} h\right) \leq U_{i, j, k}^{p+1}, \\
& U_{i, j, k}^{p+1} \leq G\left(U_{i, j, k}^{p}, U_{i, j, k}^{p}+\tilde{\Lambda} h, U_{i, j, k}^{p}+\tilde{\Lambda} h, U_{i, j, k}^{p}+\tilde{\Lambda} h, U_{i, j, k}^{p}+\tilde{\Lambda} h\right) .
\end{aligned}
$$

From (4.5), we see that

$$
-\Delta t g(-\tilde{\Lambda}, \tilde{\Lambda},-\tilde{\Lambda}, \tilde{\Lambda}) \leq U_{i, j, k}^{p+1}-U_{i, j, k}^{p} \leq-\Delta t g(\tilde{\Lambda},-\tilde{\Lambda}, \tilde{\Lambda},-\tilde{\Lambda}) .
$$

This yields (4.12) with $K^{\prime}=\max (|g(-\tilde{\Lambda}, \tilde{\Lambda},-\tilde{\Lambda}, \tilde{\Lambda})|,|g(\tilde{\Lambda},-\tilde{\Lambda}, \tilde{\Lambda},-\tilde{\Lambda})|)$. 


\subsection{Examples}

\subsubsection{A Godunov scheme}

The equation $\frac{\partial u}{\partial t}+\left|D_{H} u\right|=0$. We first consider the simpler case when $\Phi$ is the identity. We choose the Godunov like scheme proposed in [25], see also [27], which is connected with the Engquist-Osher scheme for conservation laws, see [16]. It is given by (4.4), with (4.5) and

$$
g\left(u_{1}, u_{2}, v_{1}, v_{2}\right)=\left(\min \left(u_{1}, 0\right)^{2}+\max \left(u_{2}, 0\right)^{2}+\min \left(v_{1}, 0\right)^{2}+\max \left(v_{2}, 0\right)^{2}\right)^{\frac{1}{2}} .
$$

From the inequality: for any $x \in \mathbb{R}^{4},\left(\sum_{i=1}^{4}\left|x_{i}\right|\right)^{2} \leq 4 \sum_{i=1}^{4}\left|x_{i}\right|^{2}$, and after some algebra, we see that the scheme is monotone if $2 \Delta t \leq h$.

The general case of equation (1.1). Take equation (1.1) with $\Phi$ satisfying Assumptions 3.1. Now the scheme reads (4.4), with (4.5) and

$$
g\left(u_{1}, u_{2}, v_{1}, v_{2}\right)=\Phi\left(\left(\min \left(u_{1}, 0\right)^{2}+\max \left(u_{2}, 0\right)^{2}+\min \left(v_{1}, 0\right)^{2}+\max \left(v_{2}, 0\right)^{2}\right)^{\frac{1}{2}}\right) .
$$

From the hypothesis on $\Phi$, we see that the scheme is monotone on $[-\Lambda, \Lambda]$ if $1-\frac{2 \Delta t}{h} \Phi^{\prime}(2 \Lambda) \geq 0$.

\subsubsection{The Lax-Friedrichs scheme}

The analogue of the Lax-Friedrichs scheme for equation (1.1) is (4.4), with (4.5) and

$$
g\left(u_{1}, u_{2}, v_{1}, v_{2}\right)=\Phi\left(\left(\left(\frac{u_{1}+u_{2}}{2}\right)^{2}+\left(\frac{v_{1}+v_{2}}{2}\right)^{2}\right)^{\frac{1}{2}}\right)-\theta \frac{h}{\Delta t}\left(u_{1}-u_{2}+v_{1}-v_{2}\right),
$$

where $\theta$ is a positive constant. It can be verified that the scheme is monotone on $[-\Lambda, \Lambda]$ if $0<\theta<\frac{1}{4}$ and $\theta-\frac{\Delta t}{2 h} \Phi^{\prime}(\sqrt{2} \Lambda) \geq 0$.

\section{ERror estimate}

\subsection{The main result}

We now give the main theorem:

Theorem 5.1. Under the following assumptions:

1. $\Phi$ satisfies Assumption 3.1;

2. the difference scheme (4.4) is in the form (4.5), monotone on $[-\Lambda, \Lambda]$ and consistent with (1.1);

3. the function $u_{0}$ satisfies the assumptions in point 9 of Proposition 3.1, and the interpolation $U^{0}$ of $u_{0}$ on the lattice $\left(i h, j h,(4 k+2 i j) h^{2}\right), i, j, k \in \mathbb{Z}$, satisfies the assumptions in point 7 of Proposition 4.1;

4. $L(T)$ defined by (3.6) satisfies $L(T)<\Lambda$;

5. the numerical Hamiltonian $g$ is locally Lipschitz continuous;

6. for a positive constant $C, \Delta t \leq C h$,

there exist two positive constants $H$ and $c$ (independent of $h$ ) such that for $h<H$,

$$
\left|U_{i, j, k}^{p}-u\left(\xi_{i, j, k}, t_{p}\right)\right| \leq c \sqrt{h}
$$

for all $0 \leq p \leq P$ and $i, j, k \in \mathbb{Z}$. 


\subsection{Proof of Theorem 5.1}

\subsubsection{General strategy and preliminary lemmas}

The strategy for proving Theorem 5.1 is similar to that of [14]. We seek to estimate

$$
\begin{aligned}
& \sup _{i, j, k \in \mathbb{Z}}\left|U_{i, j, k}^{p}-u\left(\xi_{i, j, k}, p \Delta t\right)\right| . \\
& 0 \leq p \leq P
\end{aligned}
$$

For that purpose, we will assume

$$
\sup _{\substack{i, j, k \in \mathbb{Z} \\ 0 \leq p \leq P}}\left(u\left(\xi_{i, j, k}, p \Delta t\right)-U_{i, j, k}^{p}\right)=\sigma>0,
$$

and look for an upper bound on $\sigma$. Were $\inf _{i, j, k \in \mathbb{Z}}\left(u\left(\xi_{i, j, k}, p \Delta t\right)-U_{i, j, k}^{p}\right)=-\sigma<0$, we could estimate $\sigma$ $0 \leq p \leq P$

exactly in the same way, so we have bounds from below and from above. For that, we define

$$
M=\left\|u_{0}\right\|_{L^{\infty}\left(\mathbb{R}^{3}\right)}+1 .
$$

Note that Propositions 3.1 and 4.1 above imply that

$$
|u| \leq M \text { on } Q, \quad \text { and } \quad\left\|U^{p}\right\|_{\infty} \leq M \quad 0 \leq p \leq P .
$$

For simplifying the notations, we call $Q=\mathbb{R}^{3} \times[0, T]$ and $Q^{d}=\left\{\left(\xi_{i, j, k}, p \Delta t\right), i, j, k \in \mathbb{Z}, 0 \leq p \leq P\right\}$. The main ingredient for obtaining the desired estimate will be a function $\Psi: Q \times Q^{d} \rightarrow \mathbb{R}$,

$$
\Psi(\eta, t, \xi, s)=u(\eta, t)-U_{i, j, k}^{p}+\left(5 M+\frac{\sigma}{2}\right) \beta_{\epsilon}(-\xi \oplus \eta, t-s)-\frac{\sigma(t+s)}{4 T}
$$

where $\xi=\xi_{i, j, k}, s=p \Delta t$ and $\beta_{\epsilon}(x, t)=\beta\left(\left|\frac{1}{\epsilon} \cdot x\right|_{K}, \frac{t}{\epsilon}\right)$, with $\epsilon$ is a positive real number and $\beta$ a smooth function on $\mathbb{R} \times \mathbb{R}$, satisfying

$$
\beta(0,0)=1, \quad 0 \leq \beta \leq 1, \quad \beta(r, t)=0 \quad \text { if } r^{4}+t^{4}>1
$$

Lemma 5.1. Under the assumptions of Theorem 5.1, there is a point $\left(\eta_{0}, t_{0}, \xi_{0}, s_{0}\right) \in Q \times Q^{d}$ such that

1. $\Psi\left(\eta_{0}, t_{0}, \xi_{0}, s_{0}\right) \geq \Psi(\eta, t, \xi, s), \forall(\eta, t, \xi, s) \in Q \times Q^{d} ;$

2. $\beta_{\epsilon}\left(-\xi_{0} \oplus \eta_{0}, t_{0}-s_{0}\right) \geq 3 / 5$.

Proof. The proof is a straightforward modification of that of Lemma 4.1 in [14].

Lemma 5.2. Let $\left(\eta_{0}, t_{0}, \xi_{0}, s_{0}\right)$ be the same as that in Lemma 5.1, and $L(T)$ be given by (3.6). We have

$$
(5 M+\sigma / 2)\left|\left(D_{H} \beta_{\epsilon}\right)\left(-\xi_{0} \oplus \eta_{0}, t_{0}-s_{0}\right)\right| \leq L(T),
$$

and

If $t_{0}>0$, then

$$
(5 M+\sigma / 2)\left|\partial_{3} \beta_{\epsilon}\left(-\xi_{0} \oplus \eta_{0}, t_{0}-s_{0}\right)\right| \leq L_{2} .
$$

with $K$ given by (3.11).

$$
-(5 M+\sigma / 2) D_{t} \beta_{\epsilon}\left(-\xi_{0} \oplus \eta_{0}, t_{0}-s_{0}\right) \leq K-\sigma /(4 T)
$$

If $0<t_{0}<T$, then

$$
(5 M+\sigma / 2)\left|D_{t} \beta_{\epsilon}\left(-\xi_{0} \oplus \eta_{0}, t_{0}-s_{0}\right)\right| \leq K+\sigma /(4 T) .
$$


Proof. The function

is maximized at $\eta_{0}$, so

$$
\eta \mapsto u\left(\eta, t_{0}\right)+(5 M+\sigma / 2) \beta_{\epsilon}\left(-\xi_{0} \oplus \eta, t_{0}-s_{0}\right)
$$

$$
(5 M+\sigma / 2)\left(\beta_{\epsilon}\left(-\xi_{0} \oplus \eta, t_{0}-s_{0}\right)-\beta_{\epsilon}\left(-\xi_{0} \oplus \eta_{0}, t_{0}-s_{0}\right)\right) \leq u\left(\eta_{0}, t_{0}\right)-u\left(\eta, t_{0}\right) \leq L(T)\left|-\eta_{0} \oplus \eta\right|_{K}
$$

On the other hand, choosing $\eta=\eta_{0} \oplus r_{1} e_{1} \oplus r_{2} e_{2}$, we have that

$$
\beta_{\epsilon}\left(-\xi_{0} \oplus \eta, t_{0}-s_{0}\right)-\beta_{\epsilon}\left(-\xi_{0} \oplus \eta_{0}, t_{0}-s_{0}\right)=\left(D_{H} \beta_{\epsilon}\right)\left(-\xi_{0} \oplus \eta_{0}, t_{0}-s_{0}\right) \cdot\left(-\eta_{0} \oplus \eta\right)+o\left(\left|-\eta_{0} \oplus \eta\right|_{K}\right) .
$$

The last observation and (5.11) yield (5.7).

We also know that

$$
\left(5 M+\frac{\sigma}{2}\right)\left(\beta_{\epsilon}\left(-\xi_{0} \oplus \eta_{0}+r e_{3}, t_{0}-s_{0}\right)-\beta_{\epsilon}\left(-\xi_{0} \oplus \eta_{0}, t_{0}-s_{0}\right)\right) \leq u\left(\eta_{0}, t_{0}\right)-u\left(\eta_{0}+r e_{3}, t_{0}\right) \leq L_{2} r .
$$

On the other hand

$$
\beta_{\epsilon}\left(-\xi_{0} \oplus \eta_{0}+r e_{3}, t_{0}-s_{0}\right)-\beta_{\epsilon}\left(-\xi_{0} \oplus \eta_{0}, t_{0}-s_{0}\right)=r \partial_{3} \beta_{\epsilon}\left(-\xi_{0} \oplus \eta_{0}, t_{0}-s_{0}\right)+o(r) .
$$

The last observation and (5.12) yield (5.8).

Similarly, the function $t \mapsto u(\eta, t)-(\sigma t) /(4 T)+(5 M+\sigma / 2) \beta_{\epsilon}\left(-\xi_{0} \oplus \eta, t-s_{0}\right)$ is maximized at $t_{0}$. If $t_{0}>0$, then for a small $r$,

$$
\left(5 M+\frac{\sigma}{2}\right)\left(\beta_{\epsilon}\left(-\xi_{0} \oplus \eta_{0}, t_{0}-s_{0}-r\right)-\beta_{\epsilon}\left(-\xi_{0} \oplus \eta_{0}, t_{0}-s_{0}\right)\right) \leq u\left(\eta_{0}, t_{0}\right)-u\left(\eta_{0}, t_{0}-r\right)-\frac{\sigma r}{4 T} \leq K r-\frac{\sigma r}{4 T},
$$

where the last inequality comes from (3.4). Then (5.9) follows immediately.

If $T>t_{0}>0$, then one obtains (5.10) in a similar way.

In what follows, we will choose

$$
\epsilon=h^{\frac{3}{8}}
$$

and the function $\beta$ such that there exists a smooth function $b: \mathbb{R}_{+} \rightarrow[0,1]$, with

$$
\begin{aligned}
\beta(x, t) & =b\left(|x|_{K}^{4}+t^{4}\right), & & \\
b(z) & =1-z, & & \text { if } z \leq \frac{1}{2} \\
b(z) & =0, & & \text { if } z \geq 1, \\
b(z) & \leq \frac{1}{2}, & & \text { if } z \geq \frac{1}{2} .
\end{aligned}
$$

The following formulas can be obtained by standard calculus: we take $(x, t)$ such $|x|_{K}^{4}+t^{4}<\frac{1}{2}$ :

$$
D_{H} \beta(x, t)=-4\left(\begin{array}{c}
\left(x_{1}^{2}+x_{2}^{2}\right) x_{1}+x_{2} x_{3} \\
\left(x_{1}^{2}+x_{2}^{2}\right) x_{2}-x_{1} x_{3}
\end{array}\right), \quad \text { and } \quad\left|D_{H} \beta(x, t)\right|=4|x|_{K}^{2} \sqrt{x_{1}^{2}+x_{2}^{2}}
$$

and

$$
\partial_{3} \beta(x, t)=-2 x_{3}, \quad \text { and } \quad \partial_{3}^{2} \beta(x, t)=-2 .
$$

Lemma 5.3. There exist two positive constants $\bar{h}$ and $C$ such that, for $h \leq \bar{h}$, calling $i_{0}, j_{0}, k_{0}$ the integers such that $\xi_{0}=\left(i_{0} h, j_{0} h,\left(4 k_{0}+2 i_{0} j_{0}\right) h^{2}\right)$,

$$
\begin{aligned}
& \left|\frac{1}{h}\left(\Delta_{+}^{1}\left(\beta_{\epsilon}\left(-\cdot \oplus \eta_{0}, t_{0}-s_{0}+\Delta t\right)\right)\right)_{i_{0}, j_{0}, k_{0}}+\left(D_{H} \beta_{\epsilon}\right)_{1}\left(-\xi_{0} \oplus \eta_{0}, t_{0}-s_{0}\right)\right| \leq C h^{\frac{1}{2}}, \\
& \left|\frac{1}{h}\left(\Delta_{+}^{1}\left(\beta_{\epsilon}\left(-\cdot \oplus \eta_{0}, t_{0}-s_{0}+\Delta t\right)\right)\right)_{i_{0}-1, j_{0}, k_{0}}+\left(D_{H} \beta_{\epsilon}\right)_{1}\left(-\xi_{0} \oplus \eta_{0}, t_{0}-s_{0}\right)\right| \leq C h^{\frac{1}{2}}, \\
& \left|\frac{1}{h}\left(\Delta_{+}^{2}\left(\beta_{\epsilon}\left(-\cdot \oplus \eta_{0}, t_{0}-s_{0}+\Delta t\right)\right)\right)_{i_{0}, j_{0}, k_{0}}+\left(D_{H} \beta_{\epsilon}\right)_{2}\left(-\xi_{0} \oplus \eta_{0}, t_{0}-s_{0}\right)\right| \leq C h^{\frac{1}{2}}, \\
& \left|\frac{1}{h}\left(\Delta_{+}^{2}\left(\beta_{\epsilon}\left(-\cdot \oplus \eta_{0}, t_{0}-s_{0}+\Delta t\right)\right)\right)_{i_{0}, j_{0}-1, k_{0}+i_{0}}+\left(D_{H} \beta_{\epsilon}\right)_{2}\left(-\xi_{0} \oplus \eta_{0}, t_{0}-s_{0}\right)\right| \leq C h^{\frac{1}{2}} .
\end{aligned}
$$


Also, if $t_{0}>0$, then

$$
\left|\frac{1}{\Delta t}\left(\beta_{\epsilon}\left(-\xi_{0} \oplus \eta_{0}, t_{0}-s_{0}\right)-\beta_{\epsilon}\left(-\xi_{0} \oplus \eta_{0}, t_{0}-s_{0}+\Delta t\right)\right)+D_{t} \beta_{\epsilon}\left(-\xi_{0} \oplus \eta_{0}, t_{0}-s_{0}\right)\right| \leq C h^{\frac{1}{2}} .
$$

Proof. The second point of Lemma 5.1 and the choice of $\beta$ yield that

$$
\left|-\xi_{0} \oplus \eta_{0}\right|_{K}^{4}+\left|t_{0}-s_{0}\right|^{4} \leq 2 \epsilon^{4} / 5 .
$$

Therefore, in a neighborhood of $\left(\eta_{0}, t_{0}, \xi_{0}, s_{0}\right)$, the function $(\eta, t, \xi, s) \mapsto \beta_{\epsilon}(-\xi \oplus \eta, t-s)$ coincides with $1-\frac{1}{\epsilon^{4}}\left(|-\xi \oplus \eta|^{4}+|t-s|^{4}\right)$. From this, we can compute $\left(D_{H} \beta_{\epsilon}\right)\left(-\xi_{0} \oplus \eta_{0}, t_{0}-s_{0}\right)$ from (5.15), and see that

$$
\begin{aligned}
\left|\left(D_{H} \beta_{\epsilon}\right)\left(-\xi_{0} \oplus \eta_{0}, t_{0}-s_{0}\right)\right|^{2} & =16 \epsilon^{-8}\left(\left(\xi_{0,1}-\eta_{0,1}\right)^{2}+\left(\xi_{0,2}-\eta_{0,2}\right)^{2}\right)\left|\xi_{0}-\eta_{0}\right|_{K}^{4}, \\
\left|\partial_{3} \beta_{\epsilon}\left(-\xi_{0} \oplus \eta_{0}, t_{0}-s_{0}\right)\right|^{2} & =4 \epsilon^{-8}\left|\xi_{0,3}-\eta_{0,3}+2\left(\eta_{0,2} \xi_{0,1}-\eta_{0,1} \xi_{0,2}\right)\right|^{2} .
\end{aligned}
$$

Then, (5.7) and (5.8) yield that

$$
\begin{aligned}
\left(\xi_{0,1}-\eta_{0,1}\right)^{2}+\left(\xi_{0,2}-\eta_{0,2}\right)^{2} & \leq\left(\frac{L(T) \epsilon^{4}}{20 M+2 \sigma}\right)^{\frac{2}{3}} \\
\left|\xi_{0,3}-\eta_{0,3}+2\left(\eta_{0,2} \xi_{0,1}-\eta_{0,1} \xi_{0,2}\right)\right| & \leq \frac{L_{2}}{10 M+\sigma} \epsilon^{4} .
\end{aligned}
$$

To summarize, as $\epsilon \rightarrow 0$, we have

$$
\begin{aligned}
\left(\xi_{0,1}-\eta_{0,1}\right)^{2}+\left(\xi_{0,2}-\eta_{0,2}\right)^{2} & \lesssim \epsilon^{\frac{8}{3}} \\
\left|\xi_{0,3}-\eta_{0,3}+2\left(\eta_{0,2} \xi_{0,1}-\eta_{0,1} \xi_{0,2}\right)\right| & \lesssim \epsilon^{4} .
\end{aligned}
$$

Let us focus on the first inegality in (5.17), because the other three are obtained in the same manner. The first thing is to notice that

$$
-\xi_{i_{0}+1, j_{0}, k_{0}} \oplus \eta_{0}=-\xi_{i_{0}, j_{0}, k_{0}} \oplus \eta_{0} \oplus\left(-h e_{1}\right)+4 h\left(\eta_{0,2}-\xi_{0,2}\right) e_{3},
$$

and that $\eta_{0,2}-\xi_{0,2}=O\left(\epsilon^{\frac{4}{3}}\right)$, because of (5.22). Thus

$$
-\xi_{i_{0}+1, j_{0}, k_{0}} \oplus \eta_{0}=-\xi_{i_{0}, j_{0}, k_{0}} \oplus \eta_{0} \oplus\left(-h e_{1}\right)+\lambda e_{3},
$$

where $\lambda=O\left(\epsilon^{\frac{4}{3}}\right) h$. Thus,

$$
\begin{aligned}
& \frac{1}{h}\left(\Delta_{+}^{1}\left(\beta_{\epsilon}\left(-\cdot \oplus \eta_{0}, t_{0}-s_{0}+\Delta t\right)\right)\right)_{i_{0}, j_{0}, k_{0}}+\left(D_{H} \beta_{\epsilon}\right)_{1}\left(-\xi_{0} \oplus \eta_{0}, t_{0}-s_{0}\right)=I+I I, \\
& I=\frac{1}{h}\left(\beta_{\epsilon}\left(-\xi_{i_{0}+1, j_{0}, k_{0}} \oplus \eta_{0}, t_{0}-s_{0}+\Delta t\right)-\beta_{\epsilon}\left(-\xi_{i_{0}, j_{0}, k_{0}} \oplus \eta_{0} \oplus\left(-h e_{1}\right), t_{0}-s_{0}+\Delta t\right)\right), \\
& I I=\left(\begin{array}{l}
\frac{1}{h}\left(\beta_{\epsilon}\left(-\xi_{0} \oplus \eta_{0} \oplus\left(-h e_{1}\right), t_{0}-s_{0}+\Delta t\right)-\beta_{\epsilon}\left(-\xi_{0} \oplus \eta_{0}, t_{0}-s_{0}+\Delta t\right)\right) \\
+\left(D_{H} \beta_{\epsilon}\right)_{1}\left(-\xi_{0} \oplus \eta_{0}, t_{0}-s_{0}\right) .
\end{array}\right) .
\end{aligned}
$$

In order to estimate $I$, we first observe that if $h$ and $\Delta t$ are small enough, then the straight line segment $\left[\left(-\xi_{i_{0}+1, j_{0}, k_{0}} \oplus \eta_{0}, t_{0}-s_{0}+\Delta t\right),\left(-\xi_{i_{0}, j_{0}, k_{0}} \oplus \eta_{0} \oplus\left(-h e_{1}\right), t_{0}-s_{0}+\Delta t\right)\right]$ is contained in the region $|x|_{K}^{4}+t^{4} \leq \frac{1}{2} \epsilon^{4}$. A first order Taylor expansion yields that $I=\frac{\lambda}{h} \partial_{3} \beta_{\epsilon}\left(\theta, t_{0}-s_{0}+\Delta t\right)$ where $\theta$ lies in the above mentioned line segment. Therefore $|I|=\frac{2|\lambda|\left|\theta_{3}\right|}{h}$, which yields that $|I| \lesssim \frac{\left(\epsilon^{4}+h h^{\frac{4}{3}}\right)}{\epsilon^{4}} \epsilon^{\frac{4}{3}} \lesssim \epsilon^{\frac{4}{3}}=h^{\frac{1}{2}}$. 
In order to estimate $I I$, we first observe that if $h$ and $\Delta t$ are small enough, then $\left\{\left(-\xi_{0} \oplus \eta_{0} \oplus \lambda h e_{1}, t_{0}-\right.\right.$ $\left.\left.s_{0}+\Delta t\right), \lambda \in[0,1]\right\}$ is contained in the region $|x|_{K}^{4}+t^{4} \leq \frac{1}{2}$. Therefore $I I=\frac{1}{h}\left(\beta_{\epsilon}\left(-\xi_{0} \oplus \eta_{0} \oplus\left(-h e_{1}\right), t_{0}-s_{0}\right)-\right.$ $\left.\beta_{\epsilon}\left(-\xi_{0} \oplus \eta_{0}, t_{0}-s_{0}\right)\right)+\left(D_{H} \beta_{\epsilon}\right)_{1}\left(-\xi_{0} \oplus \eta_{0}, t_{0}-s_{0}\right)$. A second order Taylor expansion yields that $|I I|$ is less that $c \frac{h\left(\epsilon^{\frac{8}{3}}+h \epsilon^{\frac{4}{3}}+h^{2}\right)}{\epsilon^{4}} \lesssim h^{\frac{1}{2}}$.

At this point, we have proved (5.17). In order to prove (5.18), we distinguish the case when $0<t_{0}<T$ and the case when $t_{0}=T$.

If $0<t_{0}<T$, we obtain from (5.10), (5.19) and the definition of $b$, that

$$
\left|t_{0}-s_{0}\right| \lesssim \epsilon^{\frac{4}{3}}
$$

This implies that for $h$ small enough, the line segment $\left[-\xi_{0} \oplus \eta_{0}, t_{0}-s_{0},-\xi_{0} \oplus \eta_{0}, t_{0}-s_{0}+\Delta t\right]$ is contained in the region $|x|_{K}^{4}+t^{4} \leq \frac{1}{2} \epsilon^{4}$. In that region $D_{t}^{2} \beta_{\epsilon}(x, t)=-12 t^{2} \epsilon^{-4}$. A second order Taylor expansion in $t$ yields that

$$
\begin{aligned}
& \frac{1}{\Delta t}\left(\beta_{\epsilon}\left(-\xi_{0} \oplus \eta_{0}, t_{0}-s_{0}\right)-\beta_{\epsilon}\left(-\xi_{0} \oplus \eta_{0}, t_{0}-s_{0}+\Delta t\right)\right)+D_{t} \beta_{\epsilon}\left(-\xi_{0} \oplus \eta_{0}, t_{0}-s_{0}\right) \mid= \\
&\left(12\left(t_{0}-s_{0}+\tau\right)^{2} \Delta t\right) \epsilon^{-4}
\end{aligned}
$$

with $0<\tau<\Delta t$. Thus

$$
\begin{aligned}
&\left|\frac{1}{\Delta t}\left(\beta_{\epsilon}\left(-\xi_{0} \oplus \eta_{0}, t_{0}-s_{0}\right)-\beta_{\epsilon}\left(-\xi_{0} \oplus \eta_{0}, t_{0}-s_{0}+\Delta t\right)\right)+D_{t} \beta_{\epsilon}\left(-\xi_{0} \oplus \eta_{0}, t_{0}-s_{0}\right)\right| \lesssim \\
&\left(\epsilon^{\frac{8}{3}}+h \epsilon^{\frac{4}{3}}+h^{2}\right) h \epsilon^{-4} \lesssim h^{\frac{1}{2}}
\end{aligned}
$$

and (5.18) is proved.

In the case $t_{0}=T$, we use (5.9) which can be written

$$
\left(5 M+\frac{\sigma}{2}\right) \frac{\left(T-s_{0}\right)^{3}}{\epsilon^{4}} \leq K-\frac{\sigma}{4 T}
$$

which shows that $\frac{\sigma}{4 T} \leq K$ and that $0 \leq T-s_{0} \lesssim \epsilon^{\frac{4}{3}}$. Then (5.18) is proved exactly as above.

There are now several cases to be considered, namely

- $t_{0}, s_{0}>0$

- $t_{0} \geq 0, s_{0}=0$

- $t_{0}=0, s_{0}>0$.

5.2.2. The case when $t_{0}>0, s_{0}>0$

The point $\left(\eta_{0}, t_{0}\right)$ is a maximum of the function

$$
(\eta, t) \mapsto u(\eta, t)+\left(5 M+\frac{\sigma}{2}\right) \beta_{\epsilon}\left(-\xi_{0} \oplus \eta, t-s_{0}\right)-\frac{\sigma\left(t+s_{0}\right)}{4 T} .
$$

By the definition of the viscosity solution of (1.1), we have

$$
\frac{\sigma}{4 T}-\left(5 M+\frac{\sigma}{2}\right) D_{t} \beta_{\epsilon}\left(-\xi_{0} \oplus \eta_{0}, t_{0}-s_{0}\right)+\Phi\left(\left(5 M+\frac{\sigma}{2}\right)\left|\left(D_{H} \beta_{\epsilon}\right)\left(-\xi_{0} \oplus \eta_{0}, t_{0}-s_{0}\right)\right|\right) \leq 0 .
$$

The analogous estimate on the discrete side is obtained as follows: $\left(\xi_{0}, s_{0}\right)\left(\xi_{0}=\xi_{i_{0}, j_{0}, k_{0}}, s_{0}=p_{0} \Delta t\right)$ minimizes

$$
(i, j, k, p) \mapsto U_{i, j, k}^{p}-\left(5 M+\frac{\sigma}{2}\right) \beta_{\epsilon}\left(-\xi_{i, j, k} \oplus \eta_{0}, t_{0}-p \Delta t\right)+\frac{\sigma\left(t_{0}+p \Delta t\right)}{4 T} .
$$


Thus

$$
U_{i, j, k}^{p} \geq U_{i_{0}, j_{0}, k_{0}}^{p_{0}}+\frac{\sigma\left(p_{0}-p\right) \Delta t}{4 T}-\left(5 M+\frac{\sigma}{2}\right)\left(\beta_{\epsilon}\left(-\xi_{0} \oplus \eta_{0}, t_{0}-s_{0}\right)-\beta_{\epsilon}\left(-\xi_{i, j, k} \oplus \eta_{0}, t_{0}-p \Delta t\right)\right) .
$$

Let us consider the lattice function $(i, j, k) \mapsto B_{i, j, k}$, where

$$
B_{i, j, k}=U_{i_{0}, j_{0}, k_{0}}^{p_{0}}+\frac{\sigma \Delta t}{4 T}-\left(5 M+\frac{\sigma}{2}\right)\left(\beta_{\epsilon}\left(-\xi_{0} \oplus \eta_{0}, t_{0}-s_{0}\right)-\beta_{\epsilon}\left(-\xi_{i, j, k} \oplus \eta_{0}, t_{0}-s_{0}+\Delta t\right)\right) .
$$

We aim at using (5.29) at $p=p_{0}-1$ and the monotonicity of the scheme in order to compare $U_{i_{0}, j_{0}, k_{0}}^{p_{0}}$ and $(\vec{G}(B))_{i_{0}, j_{0}, k_{0}} ;$ for this, we need to show that $\left|\left(\Delta_{+}^{1} B\right)_{i_{0}, j_{0}, k_{0}}\right|, \quad\left|\left(\Delta_{+}^{1} B\right)_{i_{0}-1, j_{0}, k_{0}}\right|, \quad\left|\left(\Delta_{+}^{2} B\right)_{i_{0}, j_{0}, k_{0}}\right|$ and $\left|\left(\Delta_{+}^{2} B\right)_{i_{0}, j_{0}-1, k_{0}+i+0}\right|$ are smaller than $\Lambda h$. But assuming $h \leq \bar{h}$, second order Taylor expansions show that this is a consequence of (5.17), (5.7) and the fact that $L(T)<\Lambda, M>1$, as soon as $h$ is small enough, say $h \leq H_{1}$. Therefore, from the monotonicity of $G$ and the fact that $U_{i, j, k}^{p_{0}-1} \geq B_{i, j, k}$ for all $i, j, k \in \mathbb{Z}$, we deduce that

$$
U_{i_{0}, j_{0}, k_{0}}^{p_{0}} \geq(\vec{G}(B))_{i_{0}, j_{0}, k_{0}},
$$

which is equivalent to

$$
\begin{aligned}
U_{i_{0}, j_{0}, k_{0}}^{p_{0}} \geq U_{i_{0}, j_{0}, k_{0}}^{p_{0}}+\frac{\sigma \Delta t}{4 T}-\left(5 M+\frac{\sigma}{2}\right)\left(\beta_{\epsilon}\left(-\xi_{0} \oplus \eta_{0}, t_{0}-s_{0}\right)-\beta_{\epsilon}\left(-\xi_{0} \oplus \eta_{0}, t_{0}-s_{0}+\Delta t\right)\right) \\
-\Delta t g\left(\begin{array}{l}
\frac{5 M+\frac{\sigma}{2}}{h}\left(\Delta_{+}^{1}\left(\beta_{\epsilon}\left(-\cdot \oplus \eta_{0}, t_{0}-s_{0}+\Delta t\right)\right)\right)_{i_{0}, j_{0}, k_{0}}, \\
\frac{5 M+\frac{\sigma}{2}}{h}\left(\Delta_{+}^{1}\left(\beta_{\epsilon}\left(-\cdot \oplus \eta_{0}, t_{0}-s_{0}+\Delta t\right)\right)\right)_{i_{0}-1_{,}, j_{0}, k_{0}}, \\
\frac{5 M+\frac{\sigma}{2}}{h}\left(\Delta_{+}^{2}\left(\beta_{\epsilon}\left(-\cdot \oplus \eta_{0}, t_{0}-s_{0}+\Delta t\right)\right)\right)_{i_{0}, j_{0}, k_{0}}, \\
\frac{5 M+\frac{\sigma}{2}}{h}\left(\Delta_{+}^{2}\left(\beta_{\epsilon}\left(-\cdot \oplus \eta_{0}, t_{0}-s_{0}+\Delta t\right)\right)\right)_{i_{0}, j_{0}-1, k_{0}+i_{0}}
\end{array}\right)
\end{aligned}
$$

Going back to (5.30), we replace each finite difference in the arguments of $g$ by the corresponding coordinate of $-\left(5 M+\frac{\sigma}{2}\right)\left(D_{H} \beta_{\epsilon}\right)\left(-\xi_{0} \oplus \eta_{0}, t_{0}-s_{0}\right)$, thereby creating errors that can be estimated in terms of $h^{\frac{1}{2}}$, thanks to (5.17) and the locally Lipschitz character of $g$. We obtain

$$
\begin{aligned}
\frac{\sigma}{4 T} & \leq\left(5 M+\frac{\sigma}{2}\right) \frac{\beta_{\epsilon}\left(-\xi_{0} \oplus \eta_{0}, t_{0}-s_{0}\right)-\beta_{\epsilon}\left(-\xi_{0} \oplus \eta_{0}, t_{0}-s_{0}+\Delta t\right)}{\Delta t} \\
& +\Phi\left(\left|\left(5 M+\frac{\sigma}{2}\right)\left(D_{H} \beta_{\epsilon}\right)\left(-\xi_{0} \oplus \eta_{0}, t_{0}-s_{0}\right)\right|\right)+C h^{\frac{1}{2}} .
\end{aligned}
$$

Making similar arguments on the $t$-difference above, we further deduce from (5.18) that

$$
\frac{\sigma}{4 T} \leq-\left(5 M+\frac{\sigma}{2}\right) D_{t} \beta_{\epsilon}\left(-\xi_{0} \oplus \eta_{0}, t_{0}-s_{0}\right)+\Phi\left(\left|\left(5 M+\frac{\sigma}{2}\right)\left(D_{H} \beta_{\epsilon}\right)\left(-\xi_{0} \oplus \eta_{0}, t_{0}-s_{0}\right)\right|\right)+C h^{\frac{1}{2}}
$$

with a new constant $C$. Taken together, (5.32) and (5.28) yield

$$
\sigma \lesssim \sqrt{h}
$$

\subsubsection{The case when $t_{0} \geq 0$ and $s_{0}=0$}

In this case, (5.30) cannot be used. Yet, the proof of (5.33) is simpler. The estimates (5.22) and (5.24) are true, because in the proof of Lemma 5.3, we did not use the fact that $s_{0}>0$. Note that (5.24) becomes $t_{0} \lesssim \epsilon^{\frac{4}{3}}$.

We have that

$$
\sup _{Q \times Q^{d}} \psi \geq \sup _{i, j, k \in \mathbb{Z}, n \geq 0}\left(u\left(\xi_{i, j, k}, t_{n}\right)-U_{i, j, k}^{n}\right)+5 M=\sigma+5 M .
$$


From this, (3.6), (3.4) and the choice of $\beta$, we see that

$$
\begin{aligned}
5 M+\sigma & \leq \Psi\left(\eta_{0}, t_{0}, \xi_{0}, 0\right) \\
& \leq\left|u\left(\eta_{0}, t_{0}\right)-u\left(\xi_{0}, t_{0}\right)\right|+\left|u\left(\xi_{0}, t_{0}\right)-u\left(\xi_{0}, 0\right)\right|+\left(5 M+\frac{\sigma}{2}\right) \beta_{\epsilon}\left(-\xi_{0} \oplus \eta_{0}, t_{0}\right) \\
& \leq L(T)\left|-\xi_{0} \oplus \eta_{0}\right|_{K}+K t_{0}+5 M+\sigma / 2 .
\end{aligned}
$$

This yields (5.33).

\subsubsection{The case when $t_{0}=0$ and $s_{0}>0$}

In this case, we can neither use (5.28), nor (5.9) and (5.10). As for (5.34), we obtain that

$$
5 M+\sigma \leq \Psi\left(\eta_{0}, 0, \xi_{0}, s_{0}\right) \leq L(T)\left|-\xi_{0} \oplus \eta_{0}\right|_{K}+K s_{0}+5 M+\sigma / 2,
$$

which implies that

$$
\sigma \leq 2 L(T)\left|-\xi_{0} \oplus \eta_{0}\right|_{K}+2 K s_{0}
$$

To estimate $s_{0}$, we use the fact that $\Psi\left(\eta_{0}, 0, \xi_{0}, s_{0}\right) \geq \Psi\left(\eta_{0}, 0, \xi_{0}, s_{0}-\Delta t\right)$, so

$$
\begin{aligned}
-U_{i_{0}, j_{0}, k_{0}}^{p_{0}} & -\frac{\sigma}{4 T} s_{0}+\left(5 M+\frac{\sigma}{2}\right) \beta_{\epsilon}\left(-\xi_{0} \oplus \eta_{0},-s_{0}\right) \\
& \geq-U_{i_{0}, j_{0}, k_{0}}^{p_{0}-1}-\frac{\sigma}{4 T}\left(s_{0}-\Delta t\right)+\left(5 M+\frac{\sigma}{2}\right) \beta_{\epsilon}\left(-\xi_{0} \oplus \eta_{0},-s_{0}+\Delta t\right) .
\end{aligned}
$$

For $h$ small enough, we can replace $\beta_{\epsilon}(x, t)$ by $1-\left|\frac{1}{\epsilon} x\right|_{K}^{4}-\frac{t^{4}}{\epsilon^{4}}$, see (5.19), and the above estimate becomes

$$
\frac{5 M+\frac{\sigma}{2}}{\epsilon^{4}}\left(s_{0}^{4}-\left(s_{0}-\Delta t\right)^{4}\right) \leq U_{i_{0}, j_{0}, k_{0}}^{p_{0}-1}-U_{i_{0}, j_{0}, k_{0}}^{p_{0}}-\frac{\sigma}{4 T} \Delta t \leq K \Delta t .
$$

This yields that

and finally

$$
\frac{5 M+\frac{\sigma}{2}}{\epsilon^{4}} s_{0}^{2}\left(4 s_{0}-6 \theta \Delta t\right) \leq K, \quad \text { for some } 0<\theta<1,
$$

$$
s_{0} \lesssim \epsilon^{\frac{4}{3}}=\sqrt{h} .
$$

From this, (5.35) and (5.21), we deduce the desired result.

\section{NumERicAl IMPLEMENTATION}

\subsection{The initial value problem}

In what follows, we assume that the function $\Phi$ is a one to one increasing function from $\mathbb{R}_{+}$onto $\mathbb{R}_{+}$, and we present the two schemes that we have tested for approximating the solution to (1.1).

The first tested scheme is the first order one proposed in (4.4), (4.5) and (4.16). We have seen above that under a stability condition, this scheme is convergent and that it produces an error of $O\left(h^{\frac{1}{2}}\right)$.

Alternatively, we will test the second order scheme proposed in [25], see also [27]: the basic trick is to build a switch that turns itself off if a singularity is detected; otherwise, it will use a higher order approximation to the neighboring values on the grid by means of a higher order polynomial using an ENO construction (see $[20,25])$. The scheme is as follows:

$$
U_{i, j, k}^{n+1}=U_{i, j, k}^{n}-\Delta t \Phi\left(\left(\max (A, 0)^{2}+\min (B, 0)^{2}+\max (C, 0)^{2}+\min (D, 0)^{2}\right)^{\frac{1}{2}}\right),
$$


with

$$
\begin{aligned}
& A=\frac{1}{h}\left(\left(\Delta_{-}^{1} U\right)_{i, j, k}+\frac{1}{2} m\left(\left(\Delta_{-,-}^{1} U\right)_{i, j, k},\left(\Delta_{+,-}^{1} U\right)_{i, j, k}\right)\right), \\
& B=\frac{1}{h}\left(\left(\Delta_{+}^{1} U\right)_{i, j, k}+\frac{1}{2} m\left(\left(\Delta_{-,+}^{1} U\right)_{i, j, k},\left(\Delta_{+,+}^{1} U\right)_{i, j, k}\right)\right), \\
& C=\frac{1}{h}\left(\left(\Delta_{-}^{2} U\right)_{i, j, k}+\frac{1}{2} m\left(\left(\Delta_{-,-}^{2} U\right)_{i, j, k},\left(\Delta_{+,-}^{2} U\right)_{i, j, k}\right)\right), \\
& D=\frac{1}{h}\left(\left(\Delta_{+}^{2} U\right)_{i, j, k}+\frac{1}{2} m\left(\left(\Delta_{-,+}^{2} U\right)_{i, j, k},\left(\Delta_{+,+}^{2} U\right)_{i, j, k}\right)\right),
\end{aligned}
$$

where the second order finite differences are given by

$$
\begin{gathered}
\left(\Delta_{++}^{1} U\right)_{i, j, k}=U_{i+2, j, k}-2 U_{i+1, j, k}+U_{i, j, k}, \\
\left(\Delta_{--}^{1} U\right)_{i, j, k}=U_{i-2, j, k}-2 U_{i-1, j, k}+U_{i, j, k}, \\
\left(\Delta_{++}^{2} U\right)_{i, j, k}=U_{i, j+2, k-2 i}-2 U_{i, j+1, k-i}+U_{i, j, k}, \\
\left(\Delta_{--}^{2} U\right)_{i, j, k}=U_{i, j-2, k+2 i}-2 U_{i, j-1, k+i}+U_{i, j, k}, \\
\left(\Delta_{+-}^{1} U\right)_{i, j, k}=\left(\Delta_{-+}^{1} U\right)_{i, j, k}=U_{i+1, j, k}-2 U_{i, j, k}+U_{i-1, j, k}, \\
\left(\Delta_{+-}^{2} U\right)_{i, j, k}=\left(\Delta_{-+}^{2} U\right)_{i, j, k}=U_{i, j+1, k-i}-2 U_{i, j, k}+U_{i, j-1, k+i},
\end{gathered}
$$

and where the switch function $m$ is

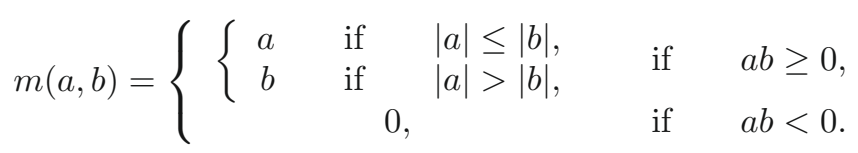

\subsection{The static Hamilton-Jacobi equation}

Here, we discuss the numerical methods for solving the static Hamilton-Jacobi equation

$$
\Phi\left(\left|D_{H} u\right|\right)=f, \quad \text { in } \mathbb{R}^{3} \backslash \bar{\omega}, \quad u(x)=u_{0}(x), \quad \text { in } \bar{\omega},
$$

where $\omega$ is a given subset of $\mathbb{R}^{3}$. For solving (6.6), the analogue of the Godunov scheme proposed in Section 4.2 .1 is

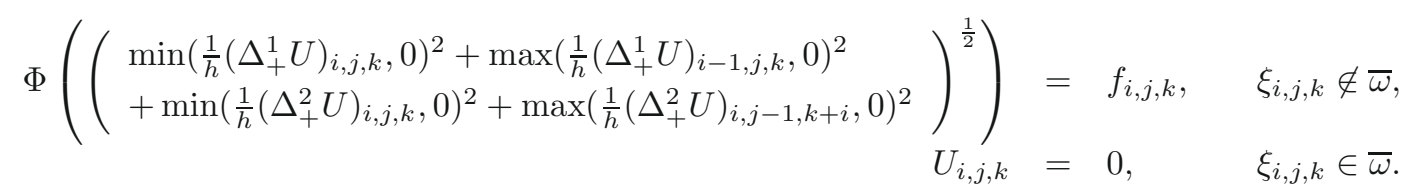

As explained in [27], a slightly different upwind scheme will turn out to be more convenient:

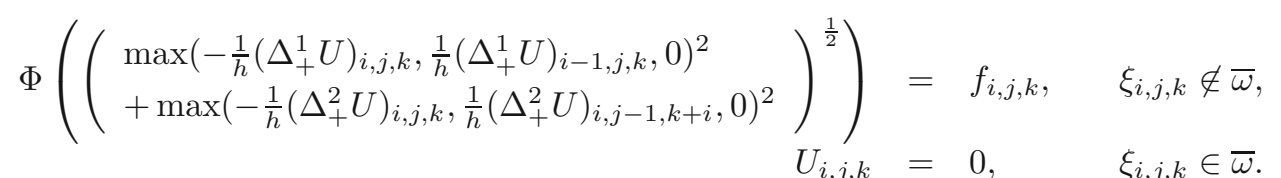

Assuming $\Phi$ is a one to one mapping from $\mathbb{R}_{+}$onto $\mathbb{R}_{+}, \Phi^{-1}\left(f_{i, j, k}\right)$ can be computed by a Newton method and the equation in (6.7) is equivalent to the quadratic equation

$$
\max \left(-\left(\Delta_{+}^{1} U\right)_{i, j, k},\left(\Delta_{+}^{1} U\right)_{i-1, j, k}, 0\right)^{2}+\max \left(-\left(\Delta_{+}^{2} U\right)_{i, j, k},\left(\Delta_{+}^{2} U\right)_{i, j-1, k+i}, 0\right)^{2}=\left(h \Phi^{-1}\left(f_{i, j, k}\right)\right)^{2} .
$$

For solving (6.7), we use the fast marching method advocated by Sethian [27]. The central idea behind it is to systematically construct $U$ using upwind values only. Indeed, the upwind difference structure of (6.7) allows us to propagate information one way, i.e. from the smaller values of $U$ to larger values. Therefore, the fast marching method consists of building the solution to (6.7) always stepping downwind: there are two zones, 
the zone where the solution is already computed or known and the zone where the solution remains to be computed. After the initialization step, the first zone only contains the boundary nodes where the solution is known, whereas the values of $U$ in the zone where the solution is not known are set to some large and positive real number. As explained in [27], we consider a thin zone of trial nodes around the existing front between the two previously mentioned zones: by and large, the fast marching method consists of the loop:

- while loop: as long as the set of trial nodes is not empty,

1. Let $\xi_{\ell, m, n}$ be the trial node corresponding to the smallest value of $U: U_{\ell, m, n}=\min \left(U_{i, j, k}\right.$ : $\xi_{i, j, k}$ is a trial node).

2. Add $\xi_{\ell, m, n}$ to the set of nodes for which the corresponding value of $U$ is known. Remove $\xi_{\ell, m, n}$ from the set of trial nodes.

3. All the neighbors of $\xi_{\ell, m, n}$, (i.e. the nodes $\xi_{\ell \pm 1, m, n}, \xi_{\ell, m \pm 1, n \mp \ell}$ ), for which the corresponding value of $U$ is not known yet, become trial nodes.

4. Recompute the values of $U$ at the trial nodes $\xi_{i, j, k}$ by solving the quadratic equation (6.8). It is important to realize that these new values $U_{i, j, k}$ only depend on the known values of $U$.

The reader can see [27] for the details of the implementation, in particular for the initialization of $U$ and of the trial zone, as well as for the use the min-heap data structure with backpointers to store the values of $U$.

It is possible to obtain a more accurate fast marching method by using a higher order scheme where it is possible to use already computed values: The idea is to define the boolean variables $\operatorname{switch}_{i, j, k}^{ \pm, \ell}, \ell=1,2$, by

$$
\begin{aligned}
\text { switch }_{i, j, k}^{ \pm, 1} & = \begin{cases}1 & \text { if } U_{i \pm 2, j, k} \text { and } U_{i \pm 1, j, k} \text { are known and } U_{i \pm 2, j, k} \leq U_{i \pm 1, j, k} \\
0 & \text { otherwise, }\end{cases} \\
\text { switch }_{i, j, k}^{ \pm, 2} & = \begin{cases}1 & \text { if } U_{i, j \pm 2, k \mp 2 i} \text { and } U_{i, j \pm 1, k \mp i} \text { are known and } U_{i, j \pm 2, k \mp 2 i} \leq U_{i, j \pm 1, k \mp i}, \\
0 & \text { otherwise. }\end{cases}
\end{aligned}
$$

With $\left(\Delta_{++}^{1} U\right)_{i, j, k},\left(\Delta_{--}^{1} U\right)_{i, j, k},\left(\Delta_{++}^{2} U\right)_{i, j, k},\left(\Delta_{--}^{2} U\right)_{i, j, k}$ the second order finite differences in $(6.3)$, and $I_{i, j, k}^{1}$, $I_{i, j, k}^{2}$ the two numbers

$$
\begin{aligned}
& I_{i, j, k}^{1}=\max \left(-\left(\left(\Delta_{+}^{1} U\right)_{i, j, k}-\frac{1}{2} \operatorname{switch}_{i, j, k}^{+, 1}\left(\Delta_{++}^{1} U\right)_{i, j, k}\right),\left(\Delta_{+}^{1} U\right)_{i-1, j, k}+\frac{1}{2} \operatorname{switch}_{i, j, k}^{-, 1}\left(\Delta_{--}^{1} U\right)_{i, j, k}, 0\right)^{2} \\
& I_{i, j, k}^{2}=\max \left(-\left(\left(\Delta_{+}^{2} U\right)_{i, j, k}-\frac{1}{2} \operatorname{switch}_{i, j, k}^{+, 2}\left(\Delta_{++}^{2} U\right)_{i, j, k}\right),\left(\Delta_{+}^{2} U\right)_{i, j-1, k+i}+\frac{1}{2} \operatorname{switch}_{i, j, k}^{-, 2}\left(\Delta_{--}^{2} U\right)_{i, j, k}, 0\right)^{2}
\end{aligned}
$$

the new scheme is

$$
\Phi\left(\left(\sqrt{I_{i, j, k}^{1}+I_{i, j, k}^{2}}\right) / h\right)=f_{i, j, k}
$$

This scheme attempts to use a second order stencil when the nodes are available and reverts to a first order one in the other cases. It is compatible with a fast marching method.

\section{Numerical RESUlts}

We present numerical tests for initial value problems first, then for stationary problems. Accuracy will be investigated in the second paragraph only, in the case of the eikonal equation. Indeed, the Carnot-Carathéodory distance to the origin can be computed by a semi-analytic method, and the schemes will be tested against the formulas. On the contrary, the goal of the first paragraph is rather to illustrate the above schemes on various cases.

\subsection{Initial value problems}

We first consider the boundary value problem (1.1)

$$
\Phi(d)=d, \quad \text { and } \quad u_{0}(x)=|x|_{K}^{2}-1 / 16 .
$$



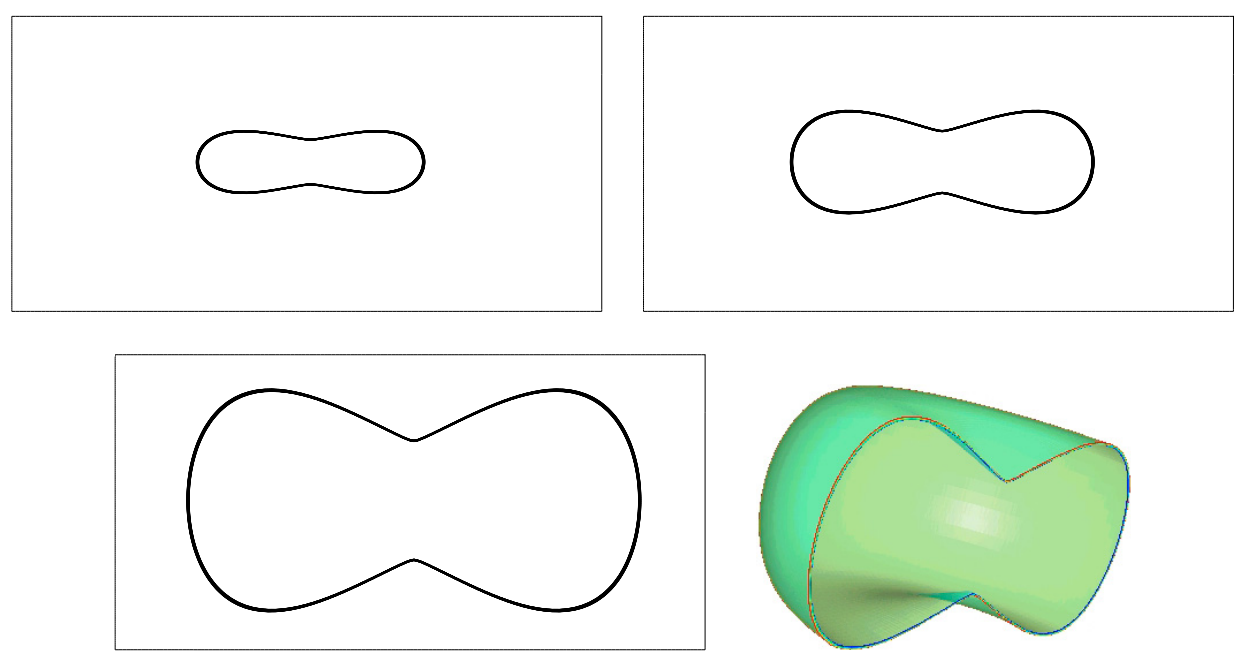

Figure 1. The solution to (1.1), with (7.1) in the plane $x_{1}=0$ at times $t=0.125, t=0.25$ and $t=0.5$ : the contour line corresponding to the value 0 . Bottom right, $3 \mathrm{D}$ view of the level set $\{x: u(x, 0.5)=0\}$ intersected with the half space $x_{1}<0$.

The viscosity solution has been approximated for $x \in(-1,1)^{2} \times\left(-\frac{1}{2}, \frac{1}{2}\right)$ and $t \in(0,1)$ with the scheme (4.4)(4.5), (4.15). The parameter $h$ is of $1 / 80$. Therefore, the lattice in the space variable has $160^{2} \times 80^{2} / 4=$ 40960000 nodes. The time step $\Delta t$ is of $1 / 320$ so the first order scheme in (4.4), (4.5), (4.15) is monotone.

Alternatively, the second-order scheme (6.1)-(6.2) in the space variable can be used.

In Figure 1, we plot the level set $\{u=0\}$ at three different times $t=0.125, t=0.25$ and $t=0.5$. The second order scheme described above has been used. The singular behavior of $u$ around the axis $x_{1}=x_{2}=0$ can be observed. As $t$ grows, the shape of the level set $\{x: u(x, t)=0\}$ becomes close to that of a Carnot sphere (see Sect. 7.2 for the description of the Carnot spheres).

The second computation is for the problem

$$
\frac{\partial u}{\partial t}+k(x)\left|D_{H} u\right|=0, \quad \text { in } \mathbb{R}^{3} \times(0, \infty), \quad u(x, 0)=|x|_{K}^{2}-1 / 16, \quad \text { in } \mathbb{R}^{3},
$$

where the Hamiltonian depends on $x$ with

$$
k(x)=1_{\left\{x_{2} \leq 0.1\right\}}+\left(1.4-4 x_{2}\right) 1_{\left\{0.1 \leq x_{2} \leq 0.3\right\}}+0.21_{\left\{0.3 \leq x_{2}\right\}} .
$$

The second order scheme has been used. The solution is displayed in Figure 2.

In the third experiment, we consider problem (1.1) with

$$
\Phi(d)=d^{2}, \quad \text { and } \quad u_{0}(x)=|x|_{K}^{2}-1 / 16 .
$$

The solution is displayed in Figure 3 at time $t=0.5$ and $t=1$. The level set $\{x: u(x, t)=0\}$ is propagated with a slower speed than in the first case.

\subsection{The eikonal equation}

Constant speed. To test the methods against semi-analytical results, we first consider the eikonal equation (2.5) for which a complete theory is available. We first aim at computing numerically the CarnotCarathéodory distance to the origin, that is the solution $u$ of problem (6.6) with $\Phi(s)=s, f=1, \bar{\omega}=\{(0,0,0)\}$ 

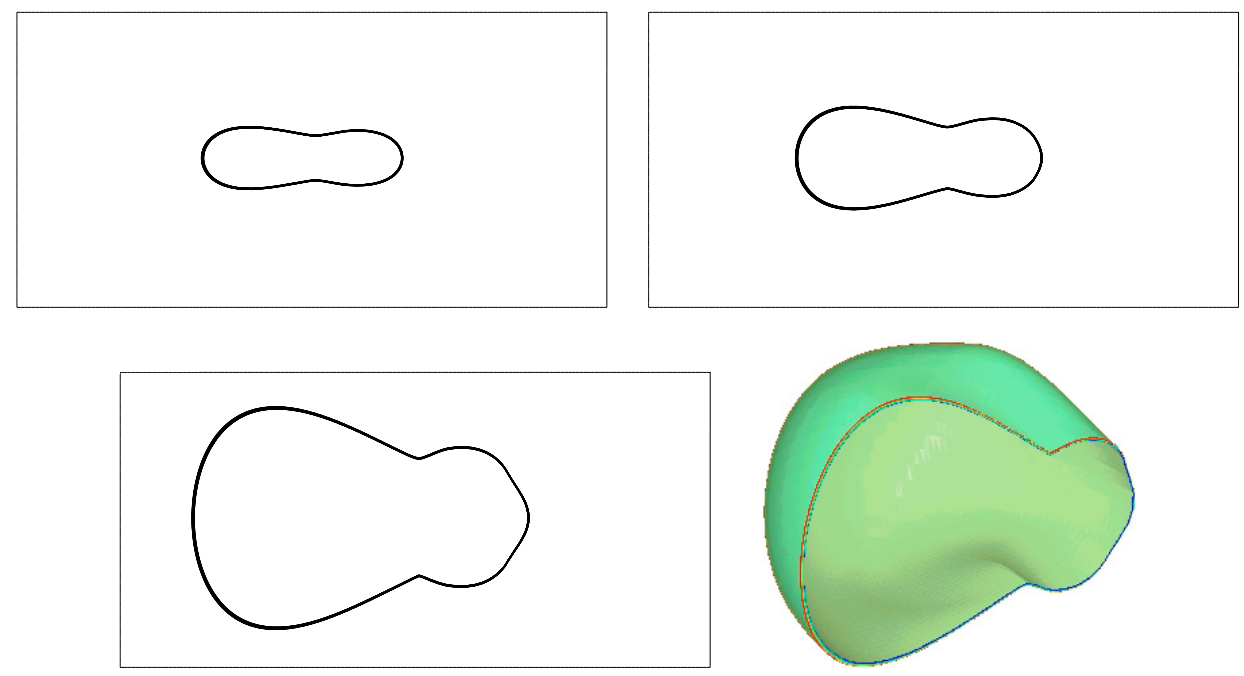

Figure 2. The solution to (7.2), with (7.3) in the plane $x_{1}=0$ at times $t=0.125, t=0.25$ and $t=0.5$ : the contour line corresponding to the value 0 . Bottom right, $3 \mathrm{D}$ view of the level set $\{x: u(x, 0.5)=0\}$ intersected with the half space $x_{1}<0$.
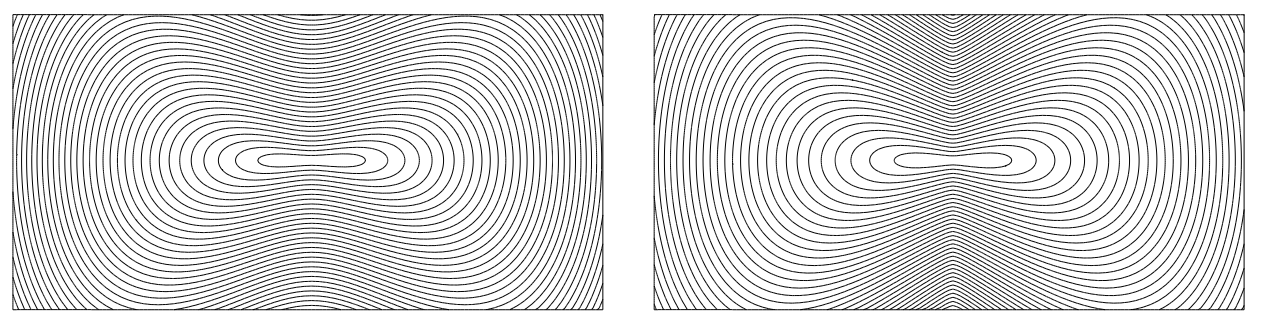

Figure 3. The solution to (1.1), with (7.4) in the plane $x_{1}=0$ at times $t=0.5$ and $t=1$ : contour lines.

and $u_{0}=0$. As shown in Beals et al. [5], the geodesics or Hamiltonian paths relative to the origin and a point $x=\left(x_{1}, x_{2}, x_{3}\right)$ such that $x_{1}^{2}+x_{2}^{2}>0$ (which satisfy $x(0)=0, x(t)=x$, for some $t>0$ ) are given by

$$
\begin{aligned}
& \left(\begin{array}{l}
x_{1}(s) \\
x_{2}(s)
\end{array}\right)=\frac{\sin (2 s \theta)}{\sin (2 t \theta)} \mathrm{e}^{(s-t) \theta \Xi}\left(\begin{array}{l}
x_{1} \\
x_{2}
\end{array}\right), \quad \text { with } \Xi=\left(\begin{array}{ll}
0 & 2 \\
-2 & 0
\end{array}\right), \\
& x_{3}-x_{3}(s)=\frac{4(t-s) \theta-\sin (2 t \theta)+\sin (2 s \theta)}{2 \sin ^{2}(2 t \theta)}\left(x_{1}^{2}+x_{2}^{2}\right),
\end{aligned}
$$

where $\theta$ is a solution to

$$
\mu(2 t \theta)=x_{3} /\left(x_{1}^{2}+x_{2}^{2}\right)
$$

and where we have set

$$
\mu(\phi)=\frac{\phi}{\sin ^{2} \phi}-\cot \phi .
$$


TABLE 1. $L^{\infty}$ error between the theoretical and computed values of $d(x ; 0)$ for $x \in[-1 / 2,1 / 2]^{3}$.

\begin{tabular}{|c||c|c|c|c|c|c|}
\hline $1 / h$ & 20 & 40 & 60 & 80 & 100 & 120 \\
\hline \hline Size & $4 \times 10^{4}$ & $6.4 \times 10^{5}$ & $3.24 \times 10^{6}$ & $1.024 \times 10^{7}$ & $2.5 \times 10^{7}$ & $5.184 \times 10^{7}$ \\
\hline \hline Scheme (6.7) & 0.121287 & 0.0769367 & 0.060584 & 0.0497205 & 0.0446911 & 0.0405027 \\
\hline \hline Scheme (6.9) & 0.0996706 & 0.0499173 & 0.0361482 & 0.0286559 & 0.0244234 & 0.0218842 \\
\hline
\end{tabular}

It is proved that (7.6) has a unique solution $2 t \theta$ in the interval $[0, \pi)$, and that the square of the CarnotCarathéodory distance $d^{2}(x ; 0)$ is the action integral corresponding to the Hamiltonian curve:

$$
\begin{array}{ll}
d^{2}(x ; 0)=\frac{4 t^{2} \theta^{2}}{2 t \theta+\sin ^{2}(2 t \theta)-\sin (2 t \theta) \cos (2 t \theta)}\left(\left|x_{3}\right|+x_{1}^{2}+x_{2}^{2}\right) & \text { if } \quad \theta \neq 0, \\
d^{2}(x ; 0)=x_{1}^{2}+x_{2}^{2} & \text { if } \quad \theta=0 .
\end{array}
$$

Thus if $x_{1}^{2}+x_{2}^{2}>0$, computing $d(x ; 0)$ requires solving the one dimensional nonlinear equation $(7.6)$ in $[0, \pi)$, which can be done numerically with Newton's method for example. If, on the contrary $x_{1}^{2}+x_{2}^{2}=0$, the Carnot-Carathéodory distance is given by

$$
d(x ; 0)=\sqrt{\pi\left|x_{3}\right|} .
$$

Let $u$ be the solution to the eikonal equation $\left|D_{H} u(x)\right|=1$ for $x \neq 0$ and $u(0)=0$, then the geodesic curve joining $x$ to the origin is computed as follows:

- Set $t=u(x)$.

- Compute $X(s), s \in[0, t]$, by solving the Cauchy problem:

$$
\frac{\mathrm{d} X}{\mathrm{~d} t}(s)=-\frac{1}{\left|D_{H} u(X(s))\right|^{2}}(\sigma(X(s)))^{T} D_{H} u(X(s)) \quad 0<s<t, \quad X(0)=x .
$$

We have tested the fast marching method with the schemes (6.7) and (6.9). Table 1 contains the error $\left.\max _{\xi_{i, j, k} \in[-1 / 2,1 / 2]^{3}} \mid U_{i, j, k}-d_{(} \xi_{i, j, k} ; 0\right) \mid$ where $U$ has been computed with the fast marching method and either the first order scheme (6.7) or the first/second order scheme (6.9). The first line of the table contains the number of unknowns, i.e. $1 /\left(4 h^{4}\right)$. In Figure 4, we have plotted the error versus $h$ in logarithmic scale. We see that the error produced by scheme (6.7) behaves like $O(\sqrt{h})$, in agreement with the theory above. The error produced by scheme (6.9) is smaller, and the slope (in logarithmic scale) of the curve lies between $1 / 2$ and 1 .

In Figure 5, we have plotted some Carnot-Carathéodory spheres centered at 0, intersected with the planar region $\{0\} \times[-0.5,0.5]^{2}$ : these spheres are obtained as the level sets of $U$ computed by scheme (6.9) with $h=1 / 100$. We very well see that the spheres have a conical singularity near the axis $x_{1}=x_{2}=0$, with an angle that gets sharper as $\left|x_{3}\right|$ grows. Note that, for obvious reasons, the grid used for representing the Carnot-Carathéodory spheres is coarser than the one used for computation, and corresponds to $h=1 / 60$.

In Figure 6, we have plotted the Carnot-Carathéodory geodesic curve between the point $(0.15,0.15,0.3)$ and the origin, computed by the semi-analytic formula (7.5) or by a discrete solution to (7.10):

- the parameter $h$ is $1 / 120$;

- in (7.10) $D_{H} u$ is first approximated at the grid nodes by a second order difference formula applied to $U$, where $U$ has been computed with scheme (6.9);

- for a point $x$ not on the grid, $D_{H} u(x)$ is computed by a bilinear interpolation of the values previously computed at the grid nodes;

- a second order midpoint scheme is used for integrating (7.10).

In Figure 6, we see that the geodesic curve is well approximated by the discrete method.

In Figure 7, we have computed the Carnot-Carathéodory distance to some compact sets $\bar{\omega}$, by solving the boundary value problem (6.6) with scheme (6.9) and $h=1 / 120$. On the left of figure, we choose $\bar{\omega}$ as 


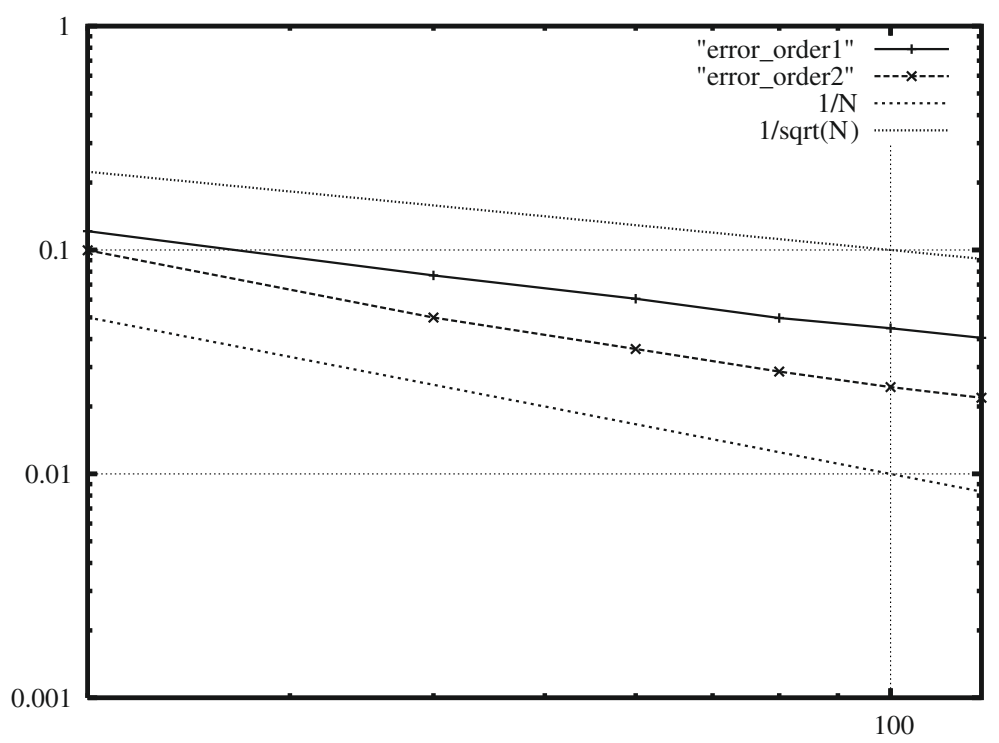

FiguRE 4. $L^{\infty}$ error between the theoretical and computed values of $d(x ; 0)$ for $x \in[-1 / 2,1 / 2]^{3}$ vs. $N=1 / h$.
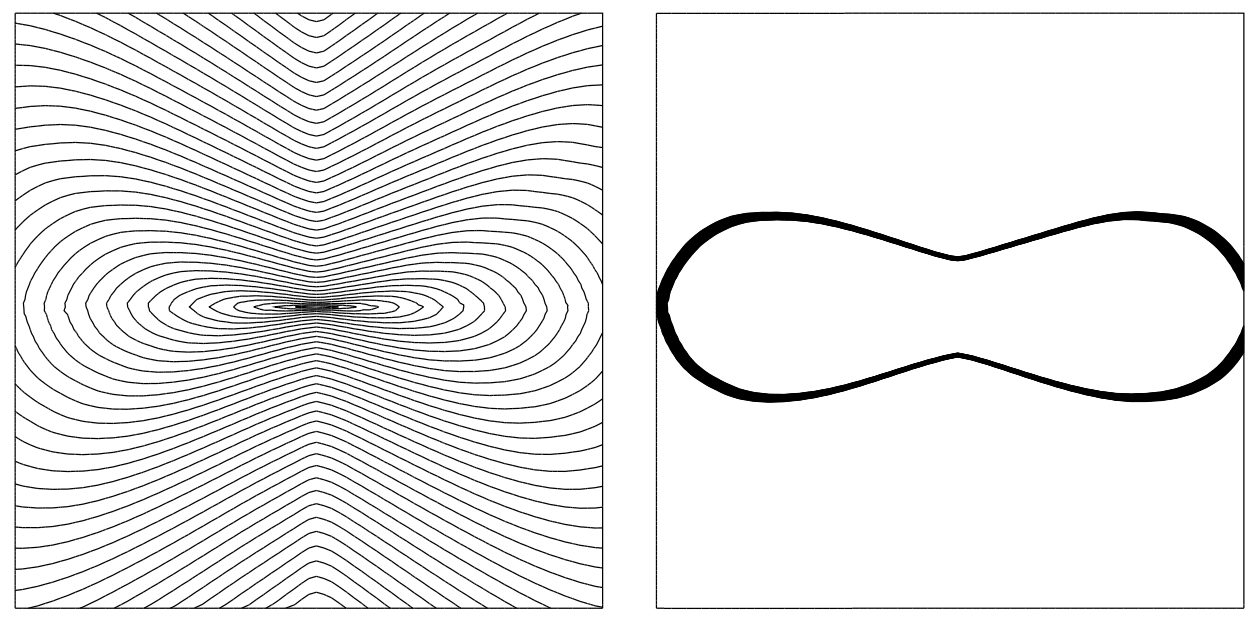

Figure 5. Left: Carnot-Carathéodory spheres $\partial B_{C}$ intersected with the plane $x_{1}=0$, found as the level sets of $U$ computed with (6.9) and $h=1 / 100$. Right: some Carnot-Carathéodory spheres with radius close to 0.5 .

the convex set $\left\{x ;\left|x_{1}\right|+\left|x_{2}\right|+\left|x_{3}\right| \leq 0.2\right\}$. On the right of the figure, $\bar{\omega}$ is nonconvex, and has the shape of a three-dimensional cross. 


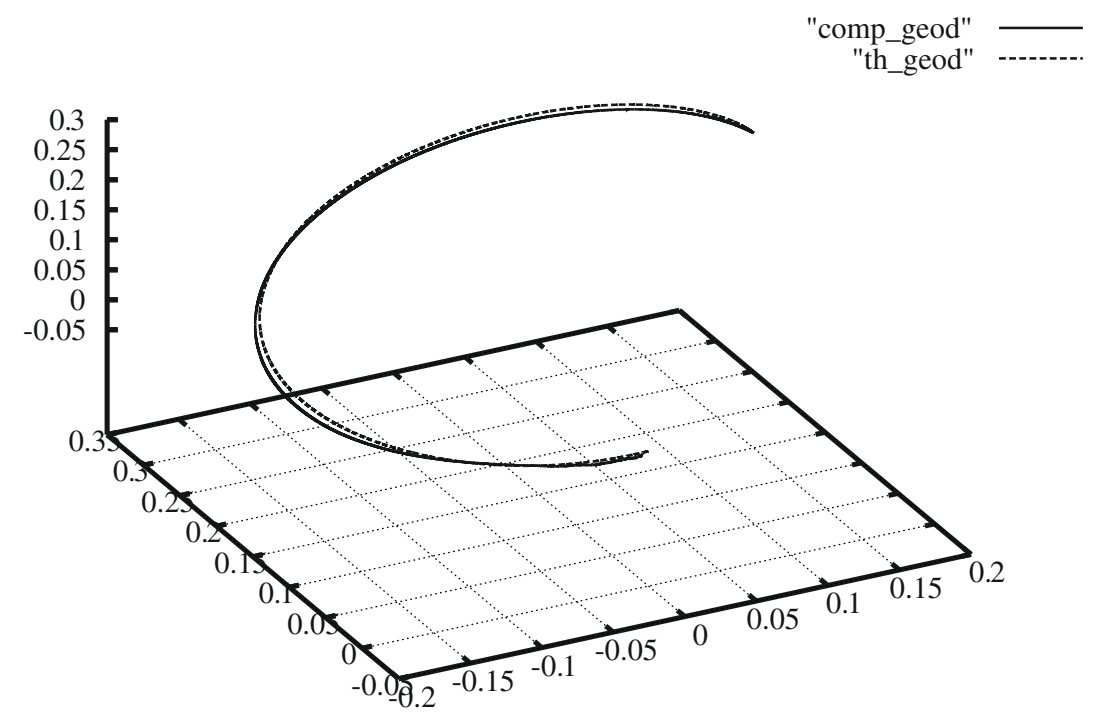

Figure 6. Comparison between the Carnot-Carathéodory geodesic joining $(0.15,0.15,0.3)$ and the origin, computed either by (7.5)-(7.6) or by (7.10), with $u$ computed by scheme (6.9) on a grid with $120 \times 120 \times \frac{\left(120^{2}\right)}{4}$ nodes.
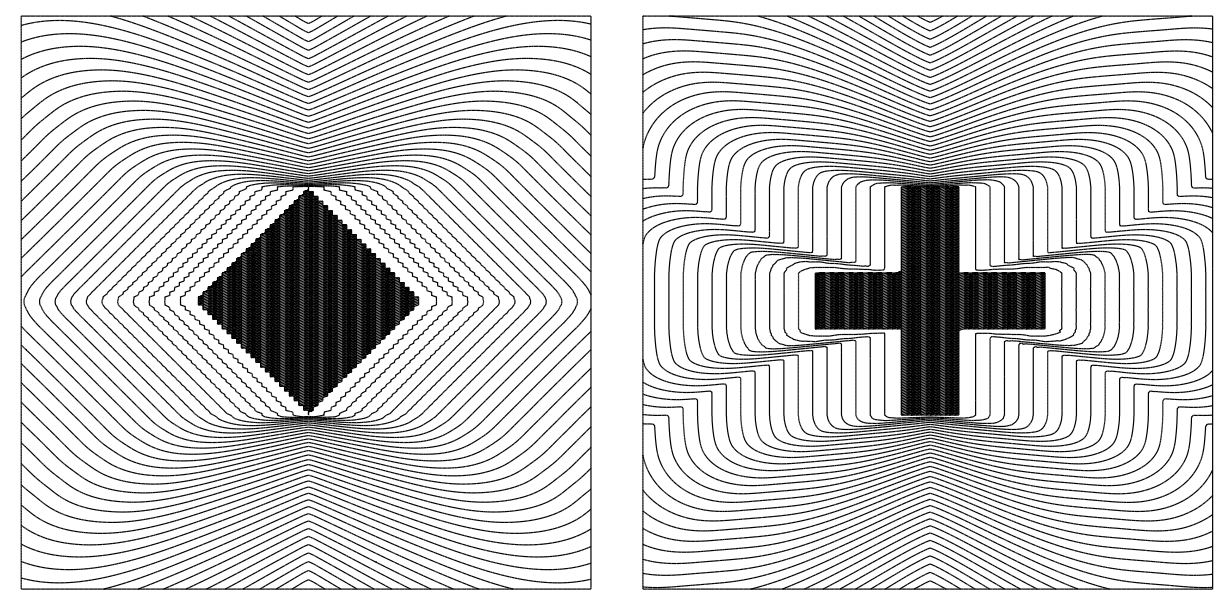

FiguRE 7. Level sets (intersected with the plane $x_{1}=0$ ) of the Carnot-Carathéodory distance to a convex set (the set $\left|x_{1}\right|+\left|x_{2}\right|+\left|x_{3}\right| \leq 0.2$ ) and to a nonconvex set.

A case with a nonuniform speed. We still solve (6.6) with $\Phi(d)=d$, but we choose

$$
f(x)=\frac{1}{\min (d(x ; 0), d(x ; A)+0.001)}, \quad u_{0}(x)=d(x ; 0),
$$

with $\omega$ is the Korànyi ball centered at the origin with radius $r=0.05$, and $A=(0,0,1 / 4)$. The contours of the solution in the plane $x_{1}=0$ is plotted in Figure 8 . 


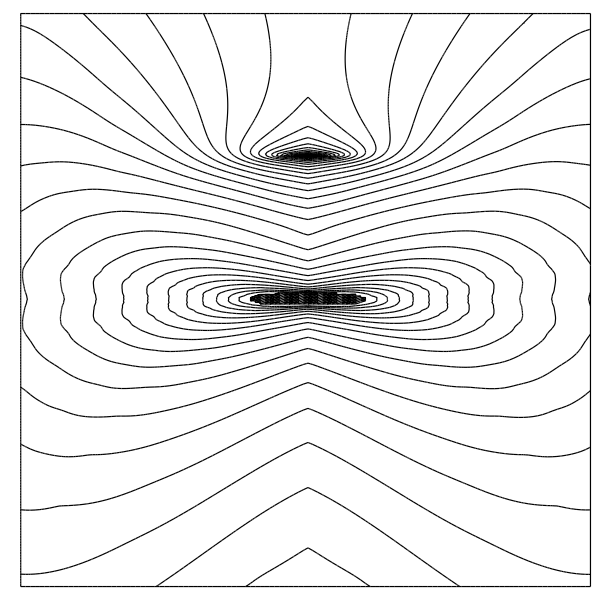

Figure 8. Contours of the solution to the static Hamilton-Jacobi equation (6.6) with (7.11), in the plane $x_{1}=0$.

\section{REFERENCES}

[1] Y. Achdou and N. Tchou, A finite difference scheme on a non commutative group. Numer. Math. 89 (2001) $401-424$.

[2] M. Bardi, A boundary value problem for the minimum-time function. SIAM J. Control Optim. 27 (1989) 776-785.

[3] M. Bardi and I. Capuzzo-Dolcetta, Optimal control and viscosity solutions of Hamilton-Jacobi-Bellman equations, Systems ES Control: Foundations \&S Applications. Birkhäuser Boston Inc., Boston, MA (1997). With appendices by M. Falcone and P. Soravia.

[4] G. Barles and E.R. Jakobsen, Error bounds for monotone approximation schemes for Hamilton-Jacobi-Bellman equations. SIAM J. Numer. Anal. 43 (2005) 540-558 (electronic).

[5] R. Beals, B. Gaveau and P.C. Greiner, Hamilton-Jacobi theory and the heat kernel on Heisenberg groups. J. Math. Pures Appl. 79 (2000) 633-689.

[6] A. Bellaïche and J.-J. Risler, Eds., Sub-Riemannian Geometry, Progress in Mathematics 144. Birkhäuser Verlag, Basel (1996).

[7] I. Birindelli and J. Wigniolle, Homogenization of Hamilton-Jacobi equations in the Heisenberg group. Commun. Pure Appl. Anal. 2 (2003) 461-479.

[8] R.W. Brockett, Control theory and singular Riemannian geometry, in New directions in applied mathematics (Cleveland, Ohio, 1980), Springer, New York (1982) 11-27.

[9] I. Capuzzo Dolcetta, On a discrete approximation of the Hamilton-Jacobi equation of dynamic programming. Appl. Math. Optim. 10 (1983) 367-377.

[10] I. Capuzzo Dolcetta, The Hopf-Lax solution for state dependent Hamilton-Jacobi equations (Viscosity solutions of differential equations and related topics) (Japanese). Sūrikaisekikenkyūsho Kōkyūroku 1287 (2002) 143-154.

[11] I. Capuzzo Dolcetta, The Hopf solution of Hamilton-Jacobi equations, in Elliptic and parabolic problems (Rolduc/Gaeta, 2001), World Sci. Publishing, River Edge, NJ (2002) 343-351.

[12] I. Capuzzo Dolcetta, A generalized Hopf-Lax formula: analytical and approximations aspects, in Geometric Control and Nonsmooth Analysis, F. Ancona, A. Bressan, P. Cannarsa, F. Clarkeă and P.R. Wolenski Eds., Series on Advances in Mathematics for Applied Sciences 76, World Scientific Publishing Co. Pte. Ltd., Hackensack, NJ (2008).

[13] I. Capuzzo Dolcetta and H. Ishii, Approximate solutions of the Bellman equation of deterministic control theory. Appl. Math. Optim. 11 (1984) 161-181.

[14] M.G. Crandall and P.-L. Lions, Two approximations of solutions of Hamilton-Jacobi equations. Math. Comp. 43 (1984) 1-19.

[15] A. Cutrí and F. Da Lio, Comparison and existence results for evolutive non-coercive first-order Hamilton-Jacobi equations. ESAIM: COCV 13 (2007) 484-502.

[16] B. Engquist and S. Osher, One-sided difference approximations for nonlinear conservation laws. Math. Comp. 36 (1981) 321-351.

[17] M. Falcone, A numerical approach to the infinite horizon problem of deterministic control theory. Appl. Math. Optim. 15 (1987) $1-13$.

[18] M. Falcone and R. Ferretti, Discrete time high-order schemes for viscosity solutions of Hamilton-Jacobi-Bellman equations. Numer. Math. 67 (1994) 315-344.

[19] S. Gottlieb, C.-W. Shu and E. Tadmor, Strong stability-preserving high-order time discretization methods. SIAM Rev. 43 (2001) 89-112 (electronic). 
[20] A. Harten, B. Engquist, S. Osher and S.R. Chakravarthy, Uniformly high-order accurate essentially nonoscillatory schemes. III. J. Comput. Phys. 71 (1987) 231-303.

[21] A. Korányi and H.M. Reimann, Quasiconformal mappings on the Heisenberg group. Invent. Math. 80 (1985) 309-338.

[22] N.V. Krylov, On the rate of convergence of finite-difference approximations for Bellman's equations with variable coefficients. Probab. Theory Relat. Fields 117 (2000) 1-16.

[23] N.V. Krylov, The rate of convergence of finite-difference approximations for Bellman equations with Lipschitz coefficients. Appl. Math. Optim. 52 (2005) 365-399.

[24] J.J. Manfredi and B. Stroffolini, A version of the Hopf-Lax formula in the Heisenberg group. Comm. Partial Diff. Eq. 27 (2002) 1139-1159.

[25] S. Osher and J.A. Sethian, Fronts propagating with curvature-dependent speed: Algorithms based on Hamilton-Jacobi formulations. J. Comput. Phys. 79 (1988) 12-49.

[26] S. Osher and C.-W. Shu, High-order essentially nonoscillatory schemes for Hamilton-Jacobi equations. SIAM J. Numer. Anal. 28 (1991) 907-922.

[27] J.A. Sethian, Level set methods and fast marching methods, Evolving interfaces in computational geometry, fluid mechanics, computer vision, and materials science, Cambridge Monographs on Applied and Computational Mathematics 3. Cambridge University Press, Cambridge, 2nd edition (1999). 